\title{
Science with Large Solar Telescopes: Overview of SpS 6
}

\author{
Gianna Cauzzi ${ }^{1}$, Alexandra Tritschler ${ }^{2}$ \\ and Yuanyong Deng ${ }^{3}$ \\ ${ }^{1}$ INAF-Ossevatorio Astrofisico di Arcetri, Firenze, Italy, \\ email: gcauzzi@arcetri.astro.it \\ ${ }^{2}$ National Solar Observatory, Sunspot, NM 88349, USA, \\ email: ali@nso.edu \\ ${ }^{3}$ Key Laboratory of Solar Activity, National Astronomical Observatories, \\ Chinese Academy of Sciences, China, \\ email: dyy@nao.cas.cn
}

\begin{abstract}
With several large aperture optical and IR telescopes just coming on-line, or scheduled for the near future, solar physics is on the verge of a quantum leap in observational capabilities. An efficient use of such facilities will require new and innovative approaches to both observatory operations and data handling.

This two-days long Special Session discussed the science expected with large solar telescopes, and started addressing the strategies necessary to optimize their scientific return. Cutting edge solar science as derived from state-of-the-art observations and numerical simulations and modeling was presented, and discussions were held on the role of large facilities in satisfying the demanding requirements of spatial and temporal resolution, stray-light correction, and spectropolarimetric accuracy. Building on the experience of recently commissioned telescopes, critical issues for the development of future facilities were discussed. These included operational issues peculiar to large telecopes as well as strategies for their best use.
\end{abstract}

Keywords. telescopes; techniques: image processing; techniques: polarimetric; methods: numerical; Sun: atmosphere, magnetic fields

\section{Introduction}

In the last decade, vast improvements have been obtained in our observational capabilities of the solar atmosphere. New spectral windows have been opened by spacebased facilities such as the highly successful Solar and Heliospheric Observatory (SOHO, Domingo et al. (1995)), Hinode (Kosugi et al. (2007)), or the recent Solar Dynamic Observatory (SDO, Pesnell et al. (2012)), avoiding the influence of Earth's atmosphere even if with reduced telescope aperture and instrument flexibility. At ground-based observatories, efficient adaptive optics systems (AO, Rimmele \& Marino (2011), Scharmer et al. (2010)) and novel instrumentation have allowed existing telescopes (e.g., VTT, DST, SST) to push the spatial resolution closer to diffraction limit from the visible to the near-infrared. Yet, when compared with realistic numerical 3D simulations of solar surface magneto-convection (e.g. Rempel (2011)), it is clear that we are still not resolving the fundamental scales at work in the solar atmosphere, which might be as small as few tens of $\mathrm{km}$ and of the order of seconds. At the same time, the critical role of the magnetic field as a main agent in shaping the solar atmosphere and its dynamics implies the need for accurate and precise high-cadence polarimetric measurements, as well as reliable 


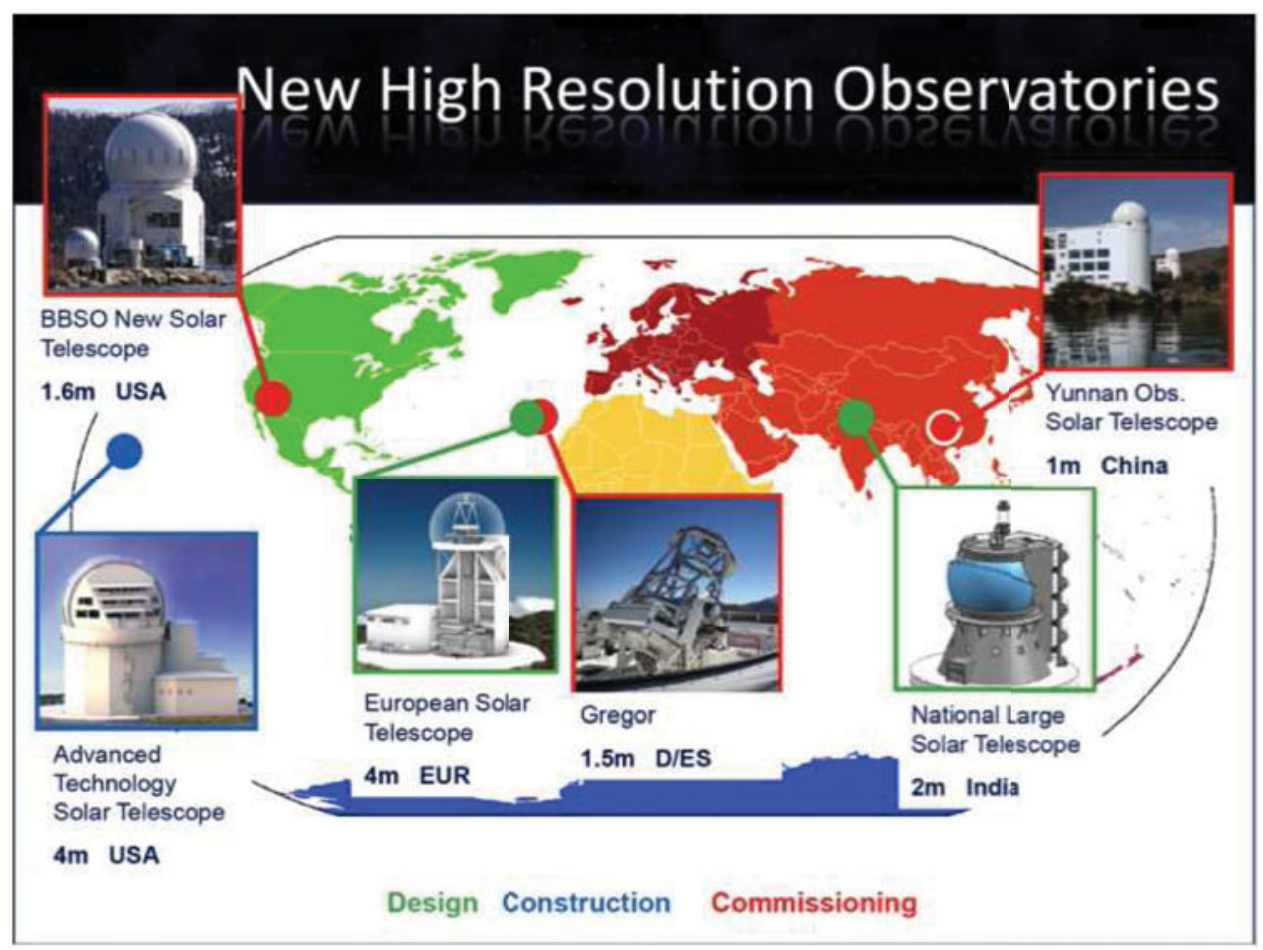

Figure 1. A world-map with the location of just commissioned, upcoming, or planned large, ground-based open solar telescopes. From Hasan (2012).

interpretational tools to obtain a quantitative knowledge of solar magnetism throughout the atmosphere.

To address these issues, in recent years a number of innovative solar telescopes, mostly working in the optical and infrared range, have been proposed by the international solar physics community to operate on the ground or from space. In particular, technological developments including the feasibility of air-cooled, open telescopes have allowed for the planning of ground-based facilities with apertures sensibly larger than the existing evacuated telescopes. These new facilities will provide for much increased spatio-temporal resolution and spectro-polarimetric sensistivity.

However, brand new challenges will accompany their operations. As an example, the foreseen use of multiple high speed, large format scientific cameras will increase enormously the data volume with respect to current standards, likely approaching hundreds of TB daily. As the pioneering efforts with the datastream of the Atmospheric Imaging Assembly (AIA, Lemen et al. (2012)) instrument on board SDO are currently showing, highly innovative solutions will be required in order to efficiently extract accurate scientific results from such large volumes (see e.g. the Heliophysics Events Knowledgebase site, at http://www.lmsal.com/hek/). Further, the scientific output of large telescopes will have to be optimized in order to justify the considerable resources invested. For ground-based telescopes, this might require developing robust data reduction pipelines to provide science-ready data to a larger user base, as well as adopting more efficient modes of operation, e.g. scheduling observations on a flexible basis in order to best match science programs to observing conditions. 
Discussion on these and other issues is just starting. In the next few years however, we expect that the operation and scientific results of new facilities, as well as instrumental upgrades in existing telescopes (including developments of Multi-Conjugate AO, chromospheric polarimetry, and tests with different types of observing modes) will yield much novel insight into the peculiarities and possibilities of observations with large solar telescopes. This Special Session, held during the XXVIII IAU General Assembly, was meant to start addressing these topics with the community at large. About 100 scientists convened for its 2-day duration, providing for a thorough and very interactive discussion on the current status and results of new telescopes, observing strategies, and critical issues for the development of future facilities that will be at the forefront of solar astrophysics in the next decades.

This paper provides a summary of the presentations and discussions held during the Session. The meeting's program is reported in the Appendix. Most of the individual presentations are available from ADS under the first author's name, with the identifier "2012IAUSS...6E".

\section{Key Scientific Questions}

Next generation large solar telescopes (see Sect. 3) are expected to make breakthrough discoveries significantly advancing our knowledge in critical solar research areas. This vital science, however, translates into significant challenges for those future endeavours since it is intricately coupled to the ability to observe the relevant solar phenomena with high precision and accuracy on unprecedented spatial scales (of the order of 10 kilometers) and time scales (of the order of seconds).

It is therefore that the Special Session 6 started with the invited introductory talk of Steiner (2012), diving into some selected solar physics problems for which small-spatial scales and low signals prevail, and extrapolating from high-resolution observations and numerical simulations to derive sensible requirements for future instrumentation. Exemplified science throughout the session focused on the following topics: photospheric and chromospheric vector-polarimetry at small spatial scales in general and the horizontal magnetic field of the quiet Sun specifically; observational evidence for a turbulent surface dynamo; drivers for dynamic fibrils and spicules; small-scale vorticity and chromospheric swirls, and large-scale coronal vortex structures. During this first session several major conclusions (or better, challenges) directly flowing from most of the science cases stood out.

First, next generation large solar telescopes should provide vectorpolarimetric observations at a spatial resolution that is well below the pressure scale height and the photon mean-free-path on the way to the dissipative scales, with an accuracy of $I_{\text {pol }} / I_{\text {cont }} \leqslant 10^{-4}$. This is most important in order to to answer remaining questions regarding the horizontal magnetic field of the quiet Sun, to reveal the structure of the hidden magnetic field, and to determine the spatial spectrum of the magnetic energy (outside active regions) with the aim of learning more about its origin and the role of the turbulent dynamo (e.g. Ishikawa (2012), Wang et al. (2012), Steiner (2012)), as well as address magnetic dissipation in the chromosphere (Judge \& Casini (2012)).

Second, it will be crucial to perfom multi-wavelength spectroscopic and spectropolarimetric observations from the photosphere up to the corona at the same time (or at least quasi-simultaneously) by creating synergies between and sensible coordination of groundbased and space-borne instruments. This capability will be increasingly important in the 
future since it should allow us to follow effects that small-scale events may have through each atmospheric layer, understand the coupling between those layers, and ultimately address the question why some stars (must) successfully sustain a net-chromosphere and coronae (e.g. Judge \& Casini (2012)). For instance, it should be possible to identify the driving mechanism and cause of dynamic fibrils (e.g. Martinez-Sykora (2012)), spicules (e.g. Klimchuk (2012)), small-scale chromospheric swirls and large-scale coronal cyclones. Specifically with regards to the latter two phenomena, it should be possible to clarify if and how those are related to or caused by small-scale vortical flows (e.g. Kitiashvili et al. (2012)) in the solar atmosphere.

It was also emphasized and became clear from discussions that diagnostic and interpretational strategies that are based on simple physical models might not be able to address the complexities of current problems in solar physics, and that a better interlocking of realistic numerical simulations with observations was necessary although not without repeated critical analysises of the numerical models (Steiner (2012), Steiner \& Rezaei (2012)). On the one hand, a solid understanding of the numerical deficiencies and the boundary and initial conditions are necessary when comparing results (e.g. in form of synthesized observables) with observations because even a so called "realistic" simulation is only a crude approximation to the reality on the Sun. Also it was pointed out that more simulations in general are needed to gain intuition with regards to the influence that changes of initial and boundary conditions have. As examples of such interlocking we refer to the invited talks of Georgobiani et al. (2012) and Martinez-Sykora (2012), and the contribution of Kitiashvili et al. (2012).

In brief, this first session, guided by the introductory presentation, took the audience from the quest for the three-dimensional structure and origin of the quiet-Sun magnetic field of the photosphere (plasma- $\beta>1$ ) upwards through the enigmatic and complex chromosphere into the corona (plasma- $\beta<1$ ) where recent observations of solar magnetized tornadoes and EUV cyclones have amazed solar observers.

\subsection{The 3D Topography and Origin of the Quiet-Sun Magnetic Field}

Since the original discovery of an omnipresent weak magnetic field outside of active regions by Livingston \& Harvey (1971) (using the Zeeman effect) at the beginning of the seventies of the last century, the magnetic field of the quiet Sun has drawn increasing attention for essentially two reasons. First, it could be a major driver of the heating of the chromosphere and ultimately the corona (e.g. Schrijver et al. (1998), Trujillo Bueno et al. (2004)). Second, its origin could be intricately coupled to both, global and local dynamo processes. In order to clarify and address those conjectures the combination of high-spatial resolution with high-precision multi-wavelength spectropolarimetry is really needed. While spectropolarimetric observations with high sensitivity in the visible and near-infrared are possible from the ground, those observations are achieved only by long integration times and as a consequence the spatial resolution (even with adaptive optics systems) is degraded to a level that significantly impedes the detection of weak fields on sub-arcsecond spatial scales.

It is thus not surprising that observations with the Spectro-Polarimeter (SP, see e.g. Lites et al. (2001)) of the Solar Optical Telescope (SOT, e.g. Kosugi et al. (2007)) on board the Hinode satellite (seeing-free environment, steady spatial resolution of $\sim 0.32$ arcsec, good polarimetric precision) considerably contributed to the advances made. This is particularly true related to the magnetic field component that is transverse to the lineof-sight (LOS) and as such detectable in the linear polarization and the Stokes- $Q$ and $U$ signals, which in the weak-field limit are intrinsically diminshed when compared to the Stokes- $V$ signal. The pre-Hinode results led to a picture where the internetwork (IN) field 
Arcseconds

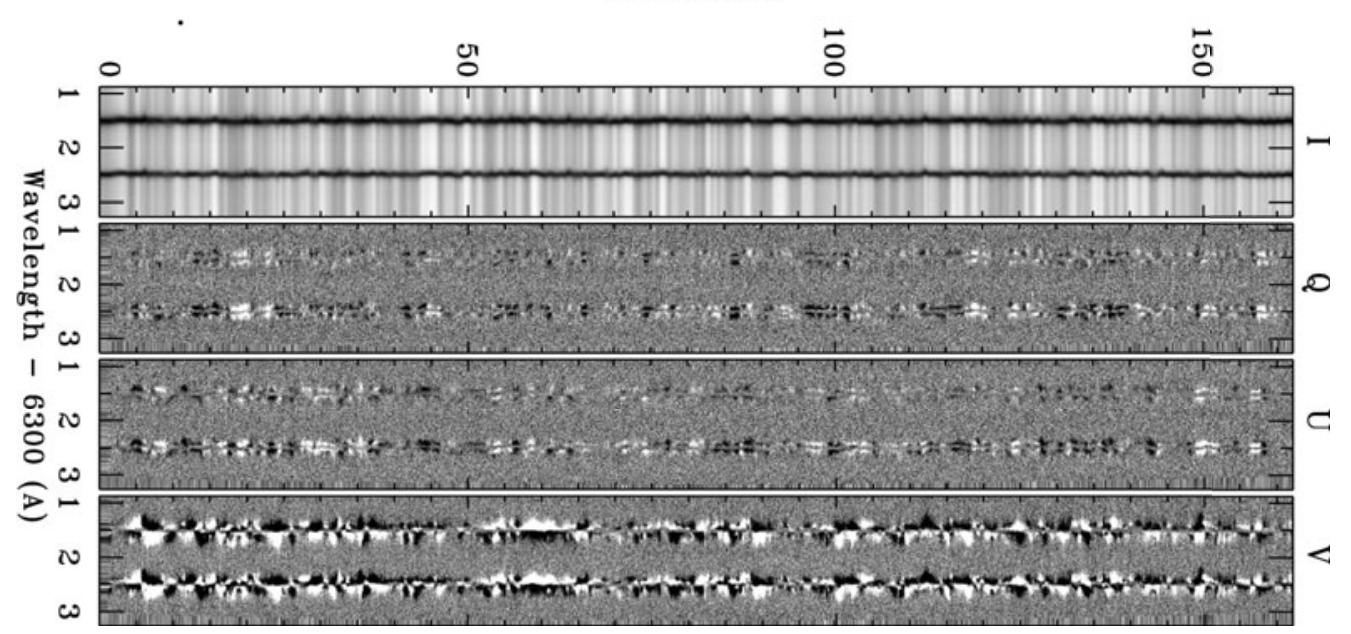

Figure 2. Typical deep mode, time-averaged Stokes $I, Q, U, V$ spectrum taken at disk center. The effective integration time is $67.2 \mathrm{sec}$ achieving a noise level in the continum of $2.9 \times 10^{-4} I_{c}$. From Lites et al. (2008).

is characterized by dynamic small-scale mixed polarities rather randomly oriented with much disputed magnetic flux densities, intrinsic field strengths, and magnetic topologies, while the network appears as a relatively organized and stable magnetic structure harbouring the stronger kilo-Gauss magnetic fields outlining the borders of the supergranulation.

The deep mode and temporally averaged Stokes spectra obtained with the SP/SOT, as visualized in Fig. 2, for the first time clearly revealed and demonstrated that the IN is heavily populated with inclined magnetic fields, evidencing a relatively large imbalance in the average apparent magnetic flux density between the vertical and horizontal component of the magnetic field (e.g. Lites et al. (2007), Orozco Suárez et al. (2007), Orozco Suárez et al. (2007), Lites et al. (2008)). It is important to remark that particularly this latter finding is much debated (see comparison of different results in Steiner (2012)). These IN transverse fields are "everywhere" (see e.g. Tsuneta et al. (2008) for a study of the Sun's polar region) and are likely the source of the seething fields discovered by Harvey et al. (2007). The total magnetic flux carried by those fields could be as high as $8 \times 10^{23} \mathrm{Mx} \mathrm{hr}^{-1}$ (e.g. Ishikawa et al. (2010)) which is higher than what sunspot regions and ephemeral regions contribute combined.

There is general agreement that IN horizontal magnetic fields are very dynamic and rather transient in nature with lifetimes in the range ot $1-10 \mathrm{~min}$, smaller than the evolutionary timescales of the granulation, and they appear with sizes that are typically smaller than a granule with a lower size limit yet un-determined (see Ishikawa (2012) and references therein). Studies of the spatial distribution of the vertical and the horizontal flux density conclude that the vertical flux prefers intergranular lanes, while the horizontal flux emerges within or at the edges of granules close to but not co-spatial with the vertical flux (see e.g. Lites et al. (2008), Ishikawa (2012), Ishikawa \& Tsuneta (2011b)). Most interestingly, vertical and horizontal flux components appear organized on mesogranular spatial scales coinciding with locations where the horizontal flow field shows a negative divergence (indicative of downflows) with "voids" in between (Lites et al. (2007), Lites et al. (2008), Ishikawa \& Tsuneta (2010a), Yelles Chaouche et al. (2011)). However, it is not clear whether (all of) these "voids" really correspond to "field free" regions 
(Ishikawa (2012)) or we just encounter the limitations with regards to sensitivity and spatial resolution of the observations (see Sect. 4.1 and contribution of Martinez Pillet (2012)). Related to the topology of the IN fields, some observations give evidence for tiny loopy structures of which some may even rise to higher atmospheric layers (e.g. Centeno et al. (2007), Martínez González et al. (2010), Ishikawa et al. (2010), Gömöry et al. (2010), Wiegelmann et al. (2010)).

The distribution of the inclination of IN magnetic fields is similarly debated as the apparent flux densities and intrinsic field strengths. No consensus has been reached so far. While numerical simulations of magneto-convection (independent of the orientation of the seed field) predict a predominance of the horizontal field increasing with height (Steiner et al. (2008), Schüssler \& Vögler (2008), Danilovic et al. (2010)), results from observations using different methodology (inversions, weak-field approximation) and wavelength regimes (visible and infrared) do not converge. Some authors find predominantly horizontal IN fields (Lites et al. (2007), Lites et al. (2008), Orozco Suárez et al. (2007), Danilovic et al. (2010)), other studies point towards predominantly vertical fields (e.g. Khomenko et al. (2003), Beck \& Rezaei (2009), Stenflo (2010)), and some find indication for (quasi) isotropic distributions (e.g. Martínez González et al. (2008), Asensio Ramos (2009), Bommier et al. (2009)).

It is argued that the different sensitivity in linear polarization and circular polarization to the magnetic field combined with the finite sensitivity of the photon noise inflicted measurements leading to selection effects (e.g. Kobel et al. (2011)), is the main reason for the many disparate results. However, Beck \& Rezaei (2009) argue that the thermodynamic state of the atmosphere and different formation heights of the lines (visible and infrared) play an important role as well. The distribution of the magnetic flux and the angular distribution are of such special interest since they can provide clues about the origin and cause of the quiet-Sun internetwork magnetism. Mostly, observational results appear consistent with the notion that the action of a local dynamo driven by turbulent convection is a contributor to the generation and maintenance of the quiet-Sun's internetwork magnetic field (e.g. Cattaneo (1999), Vögler \& Schüssler (2007), Schüssler \& Vögler (2008), Pietarila Graham et al. (2009), Pietarila Graham et al. (2010)). In this context, it is particularly conspicuous that the amount of observed IN magnetic flux neither indicates a solar-cycle nor any latitudinal dependence (e.g. Hagenaar et al. (2003), Sánchez Almeida (2003), Trujillo Bueno et al. (2004), Harvey et al. (2007)). However, it remains to be answered to which degree a surface dynamo contributes to the IN magnetism and what fraction does result from the global magnetic field. Furthermore, the detection of "voids" in the IN magnetic field needs to be better understood in the context of a local dynamo working on granular spatial scales (see also Sect. 4.1). Specifically in this area more "realistic" simulations are needed that allow a comparison between the observations and the predictions of those simulations (hopefully in form of synthesized observables). Furthermore a proper understanding of the initial and boundary conditions of the individual simulations is needed. Georgobiani et al. (2012) presented "realistic" 3D simulations of solar magnetoconvection leading to flux emergence and the formation of pores and active regions without an initial coherent flux rope implying that a tachocline is not necessary. Performing a forward synthesis of Stokes spectra, Georgobiani et al. (2012) also point out that the mere limitations imposed by a finite aperture do already significantly alter the Stokes spectra.

In summary, as much progress has been made since the launch of Hinode satellite many questions remain un-answered. Most important, what is the structure of the magnetic field on spatial scales beyond the SP/SOT 0.32 arcsec benchmark? This addresses the hidden magnetic flux issue and the discrepancies between the results when using Zeeman- 
or Hanle diagnostics. The intrinsic problem that the net-Stokes signal of co-existent opposite polarities within the same resolution element are hard to distinguish from a single polarity with small filling factor will only be solved when the spatial resolution is pushed further and further down to smaller scales. Hence, accurate and precise vectorpolarimetry of the photosphere (and chromosphere!) very close to the diffraction limit of future telescopes will play an incredible important role allowing for new discoveries and hopefully resolving some existing riddles.

\subsection{Photospheric Vorticity, Chromospheric Swirls and Coronal Tornadoes}

Another area of active research currently living through a rennaissance is concerned with small-scale vorticity in the photosphere and its consequences for the higher atmospheric layers. Vortex flows are nothing new, they have been theoretically predicted based on 3D numerical simulations of solar convection already back in the 80's by Nordlund (1985) introducing descriptive terms like bathtub effect and inverted tornado. Several observational attempts were undertaken to prove the existence of those flow structures but without success for only one exception. The first confirmation of a vortical flow although with a larger size (covering several granules, lifetime of $\sim 1.5$ hours) than predicted must be attributed to Brandt et al. (1988) suggesting that vortical flows could introduce twisting motions of the footpoints of magnetic flux tubes and as such provide a mechanism for heating the chromosphere and corona (also Balmaceda et al. (2010)).

With increasing spatial resolution various studies were later able to infer vortex flows on small spatial scales $(\leqslant 0.5 \mathrm{Mm})$ based on local correlation tracking techniques calculating horizontal flow maps and/or tracing the proper motion of photospheric magnetic elements that get "dragged into" and "trapped" in the downdraft of a vortex (e.g. Bonet et al. (2008), Bonet et al. (2010), Balmaceda et al. (2010), Vargas Domínguez et al. (2011)). Those small-scale vortical flows or whirlpools show lifetimes up to $20 \mathrm{~min}$ with varying values for the space-time density in the range $1.8-3.1 \times 10^{-3} \mathrm{Mm}^{-2}$ minute ${ }^{-1}$. The observations are not conclusive about whether a preferred sense of rotation exist. Only Bonet et al. (2010) find a preferred rotation sense using high-resolution observations obtained with the IMaX instrument of the SUNRISE balloon. Vortical flows or "vortex tubes" are not restricted to the vertical but have been identified also oriented in the horizontal direction and being closely related to the sub-structure of granules (dark lanes and bright rims) and their "edgy" appearance (Steiner et al. (2010)). However, with the current spatial resolution it remains open whether those observable "granular lanes" are just the most prominent out of a wide spectrum of vortex tubes (Steiner (2012)).

The observational evidence of photospheric vortex flows is accompanied by recent numerical simulations examining small-scale vorticity and the consequences. For instance, Kitiashvili et al. (2011) find that simulated whirlpools are quite numerous, form in intergranular lanes with no preferred sense of rotation, are characterized often by very strong horizontal shear velocities $\left(7-11 \mathrm{~km} \mathrm{sec}^{-1}\right)$, strong downflows in the vortex cores $\left(7 \mathrm{~km} \mathrm{sec}{ }^{-1}\right)$, and very low densities ("density holes"). However, the cores (where much of the motion takes place) of those simulated whirlpools are tiny in the range of $100 \mathrm{~km}$ which is challenging to spatially resolve (see Fig. 3). Most interesting, they find that their simulated whirlpool flows can attract other vortical flows with opposite vorticity which then interact with each other, partially annihilate and in the aftermath excite acoustic waves. Observational evidence for the latter has been found analyzing a time sequence of $\mathrm{TiO}$ images obtained with the New Solar Telescope (NST) of the Big Bear Solar Observatory (Kitiashvili et al. (2012)). The simulations of solar surface convection by Moll et al. (2011) provide a complex picture where regions of high "swirling strength" form an unsteady network of tangled filaments. Close to the optical surface, vertical and 

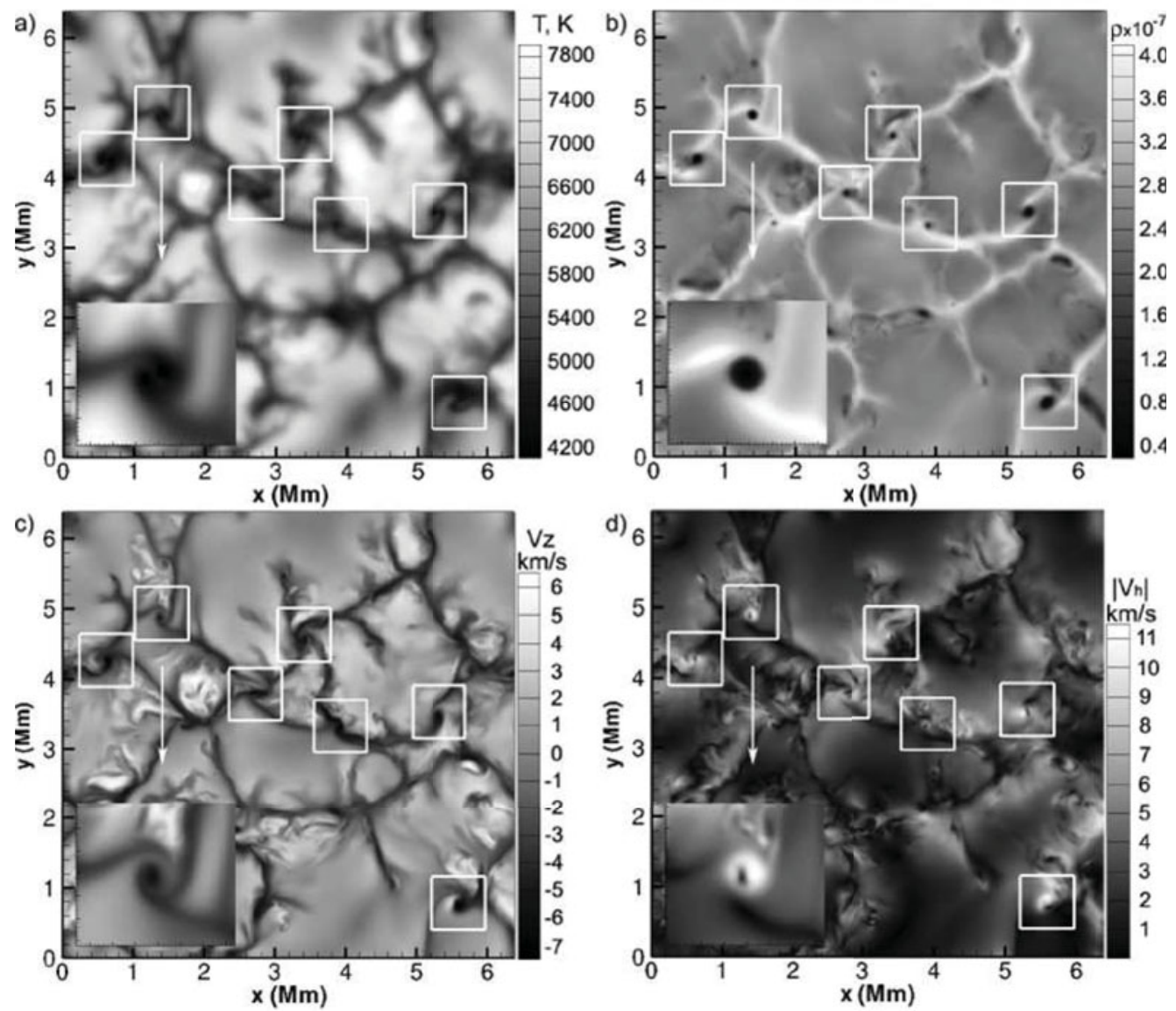

Figure 3. Snapshots of the simulation results at the solar surface for (a) temperature, (b) density, (c) vertical velocity, and (d) magnitude of the horizontal velocities. White squares indicate the largest whirlpools. From Kitiashvili et al. (2012).

horizontal swirls are preferentially found within the intergrnaular lanes and at the edges of granules, respectively. Above the surface, swirls appear in many shapes as small-scale bent and arc-like filaments with diameters of only $30 \mathrm{~km}$ which renders observational proof impossible with current solar telescopes.

It is interesting to remark that photospheric vortex flows do appear with sizes larger than just a couple of granules. For example, Attie et al. (2009) observed very long-lasting (1-2 hours) photospheric vortex flows extending up to 27 arcsec that are located at the supergranular junctions. Those observations specifically showed that opposite polarity magnetic elements co-exist in the same vortex where one magnetic element caught at the vortex core, is orbited by the opposite polarity magnetic element.

Recently, observations of the quiet chromosphere in a coronal hole demonstrated that whirling motions are not limited to the photosphere. From a time sequence of Ca II $854.21 \mathrm{~nm}$ line-core images, Wedemeyer-Böhm \& Rouppe van der Voort (2009) were able to identify numerous (although subtle) chromospheric swirls made of dark and bright rotating patches in form of arcs, spiral arms, rings or ring fragments (also Wedemeyer et al. (2013)). Those swirls typically have a width of 2 arcsec (with the fragments being much smaller, $\sim 0.2$ arcsec) and indicate fast upflows up to $7 \mathrm{~km} \mathrm{sec}{ }^{-1}$. Most interestingly, below those chromospheric swirls, groups of magnetic elements move 
with respect to each other but there is no indication for the presence of a vortical flow. Wedemeyer-Böhm et al. (2012), however, identify the chromospheric swirls as observational signatures of rapidly rotating magnetic structures ("tornadoes") rooted in the photosphere in magnetic elements and extending through the tansition region (He II $30.4 \mathrm{~nm}$ ) into the low corona (Fe IX $17.1 \mathrm{~nm}$ ). The observations are supported by numerical simulations using the CO5BOLD code (Freytag et al. (2012), Beeck et al. (2012)) suggesting that chromospheric swirls are the (chromospheric) imprint of rotating magnetic elements braiding and twisting the magnetic field. A numerical experiment comparing three different simulation runs (field-free case, vertical or horizontal seed field of $50 \mathrm{G}$ ) show that chromospheric swirls develop in the vertical run but were absent in the field-free and horizontal run (see Steiner \& Rezaei (2012)). Similarly, the simulations of Kitiashvili et al. (2012) show that the swirling motions of vertical vortex tubes can capture and twist magnetic fields forming magnetized vortex tubes penetrating into the chromosphere. The ubiquity of those structures and the fact that thy provide a direct link between the convection zone and the upper solar atmosphere suggests that they could considerably contribute to the channeling of energy into the chromosphere and corona.

In the corona and on much larger spatial scales, Zhang \& Liu (2011) and Su et al. (2012) find EUV cyclones and solar magnetized tornadoes, respectively, based on observations with the AIA instrument of the SDO. The cyclones are quite abundant and are seen everywhere on the Sun with the sense of rotation showing a weak hemispheric preference, last for several hours and are found rooted in rotating network magnetic fields. The solar magnetized tornadoes typically appear in groups, are related to filaments/prominences, and are believed to be driven by underlying photospheric vortex flows.

In summary, small-scale vorticity generated in the photosphere by convective motions interacting with the magnetic field has implications throughout the solar atmosphere and seems to be the source of a many recently discovered phenomena. However, the small sub-arcsecond spatial scales over which the actual motions take place and disturbances propagate through the atmosphere make it extremely difficult to observe those phenomena with present day solar telescopes. Observationally, vortex motions are identified by local correlation tracking techniques and feature tracking algorithms, but not with spectroscopic methods. For instance, a direct measurement of the individual rotation of a magnetic element trapped in a vortex flow and the braiding and twisting of the magnetic field has not been achieved so far and will probably need the enhanced osberving capabilities of future large telescopes.

\subsection{Chromospheric Structure and Dynamics}

The chromosphere is the focus of much contemporary research. This boundary region hosts the critical transition from fluid to magnetic dominance on the atmospheric plasma, and its many complexities (partial ionization, non-LTE, time-dependent $\mathrm{H}$ ionization, large spatial and temporal gradients, role of mechanical heating) represent a formidable challenge to our understanding of a magnetized stellar atmosphere. Yet, as a key interface between the photospheric magneto-convection "driver" and the resulting outer solar atmosphere and heliosphere, as well as the main source of the ionizing UV radiation that drives the Earth's atmosphere, it is mandatory that we unravel its working.

In a sense, the presence of a chromosphere and a corona derives fairly straightforwardly from the existence of any non-radiative heating source at the solar surface (Judge \& Casini (2012)). How this energy is actually transported at larger heights, and how it is dissipated in loco, remains however the problem in defining the solar chromospheric structure, even if observations clearly indicate that magnetism lies at the heart of the 
phenomenon. Measuring the vector magnetic field in the chromosphere and its variations in time and/or height appears as the proper way to obtain reliable estimates of the "free energy" available in the magnetic configuration for local dissipation (Judge (2009)), or a direct measure of the electric currents' density (Socas-Navarro (2005)) $\dagger$.

This is arguably a very hard task with most chromospheric signatures (e.g. Uitenbroek (2011)); still, a very promising tool to this end is the use of the He I triplet at $1083 \mathrm{~nm}$ (see also Sect. 4.2), a spectral signature showing large sensitivity to chromospheric magnetic fields through both the Hanle and Zeeman effect (Asensio Ramos et al. (2008), Schad et al. (2013)). The required polarimetric precision must be high, of the order or better that $10^{-4}$, to be able to properly measure the vector components of the field: this implies that new large aperture telescopes will have to function as large "light buckets", relaxing the requirement of diffraction-limited spatial resolution for the case of reliable chromospheric polarimetry (see Judge \& Casini (2012) and Sect. 5).

A complementary, important approach in the study of the chromosphere is that provided by 3D-MHD numerical simulations. Notwithstanding the complications of the many non-linear equations that govern the physics of the solar magnetized atmosphere, and the proper treatment of radiation and scattering (see e.g. Skartlien (2000), MartinezSykora (2012)), recent advances in this area are bringing about "realistic" simulations which encompass the whole atmosphere, and the very different physical regimes present from the convection zone to the corona (see among others, Fang et al. (2010), Gudiksen et al.(2011)). Special care must be given in the chromosphere and transition region to physical processes inherent to the low density, magnetized, highly intermittent conditions of plasma, including thermal conduction, partial ionization effects and ionneutral interaction, or non-equilibrium effects such as hydrogen ionization (Leenaarts et al. (2007), Martínez-Sykora et al. (2012), Khomenko \& Collados (2012), Leake \& Linton (2013)). Recent efforts in these directions show some very promising results about the reliability of the simulations; in particular the introduction of partial ionization (treated as multi-fluid or otherwise) in the simulations' domain has shown that the resulting Pedersen resistivity is of the same order of the numerical resistivity value normally used to stabilize the codes, an issue which had been long cause of concern (Martínez-Sykora et al. (2012), de la Cruz Rodríguez et al. (2013)). One of the most important limitations remains the limited spatial extension of the simulations, usually of the order of a super granular cell or less, which might hide important phenomena related to large scale magnetic connectivity (Martinez-Sykora (2012)).

The comparison of the time evolution of MHD variables in the simulations with observed quantities is an important tool to understand dynamic chromospheric phenomena or, conversely, to determine whether simulations are missing some important physics. Some intriguing small-scale phenomena like dynamic fibrils (De Pontieu et al. (2007)) have been successfully explained as due to magneto-acoustic shocks propagating along field lines in the upper atmosphere (Heggland et al. (2007), Martínez-Sykora et al. (2009)), even if some discussion is still ongoing about the origin of the driving piston. In particular, the simulations of Kato et al. (2011) identify the "turbulent pumping" mechanism as an alternate way to excite longitudinal waves along the magnetic flux tubes. This is an interesting example of how simulations carried out by different groups can

$\dagger$ While extrapolation of photospheric magnetic maps is often attempted, most notably to infer coronal conditions, the required assumption of force-free status for the magnetic field in the photosphere is usually not satisfied, leading to inconsistencies in the derived results, see e.g. De Rosa et al. (2009). 
provide different insights (or results) into the physics of selected phenomena, and thus of the need to properly understand the role of, e.g., different numerical methods, boundary conditions etc., as remarked above. Extreme high resolutions observations such as provided by large-aperture telescopes, in this case of downflows around small magnetic network elements, could provide clear discriminant between conflicting hypotheses.

An intense debate is currently undergoing in the community about a similar smallscale, dynamical chromospheric phenomenon, namely "type II spicules". These were first defined through Hinode Ca II observations at the limb as very thin (at the limit of telescope resolution of 0.2 arcsec, rapidly evolving (50-150 sec) features, which are observed to propagate upward (velocities of $30-100 \mathrm{~km} \mathrm{sec}^{-1}$ ) and fade around their maximum length rather than fall down to the solar surface (de Pontieu et al. (2007)). Ever-present type-II spicules have been identified as main agents in the transfer of energy and mass from the photosphere to the corona in several works (e.g. De Pontieu et al. (2009), De Pontieu etal (2011)), attributing their fading from chromospheric signatures to heating to coronal temperatures. Arguments to the contrary argue that EUV spectroscopy indicates at most a very small fraction of coronal plasma originating from such features (Klimchuk (2012), Klimchuk (2012)), and that at least a fraction of type-II spicules could be due to warps in two-dimensional sheet-like structures, related to the magnetic tangential discontinuities naturally arising in low- $\beta$ plasma (Judge et al. (2011), Judge et al. (2012)).

Numerical simulations are for now just hinting at the creation of type-II spicules, in rather particular conditions (Martinez-Sykora (2012)), a fact that seems in contrast with their prevalent appearance over the whole solar surface (Pereira et al. (2012)). Further comparisons of diagnostics synthesized from simulations adopting different magnetic topologies and spatial domains, with proper resolution observations of the chromosphere are expected to much advance our understanding of this fascinating phenomenon. Indeed, many of the technical requirements for the new, large solar telescopes derive from chromospheric science of this kind (see e.g. Sects. 3 and 4).

\section{Existing and Planned Large Facilities}

\subsection{Ground-based optical telescopes}

A large number of new ground based facilities have been proposed, constructed and commissioned in recent years; virtually all of them have been presented during the second session of SpS6, as listed in the Appendix. After many years of "stagnation" in the field, two main reasons are behind this striking development. The first one is a series of technological breakthroughs which have finally allowed the design of air-cooled, open large solar telescopes. By virtue of this design, one can plan for much larger apertures than for the case of "classical" evacuated telescopes, and aim at achieving spatial resolutions well below the current $\approx 0.2^{\prime \prime}(150 \mathrm{~km})$ limit, thus approaching some of the important spatial scales predicted by simulations. The second one is the advent of reliable and efficient Adaptive Optics systems, coupled with powerful image-reconstruction techniques (e.g. Rimmele \& Marino (2011), Wöger (2010)), both indispensable tools to achieve resolutions close to the actual diffraction limit of these large telescopes.

Facilities recently become operative, such as the German on-axis GREGOR Telescope on Tenerife (Schmidt et al. (2012), Denker et al. (2012), currently being commissioned) or the New Solar Telescope (NST, Goode \& Cao (2012), Cao (2012) of the Big Bear Solar Observatory, online since 2010), have sizes around $1.5 \mathrm{~m}$ (Fig. 4). They represent a funda- 

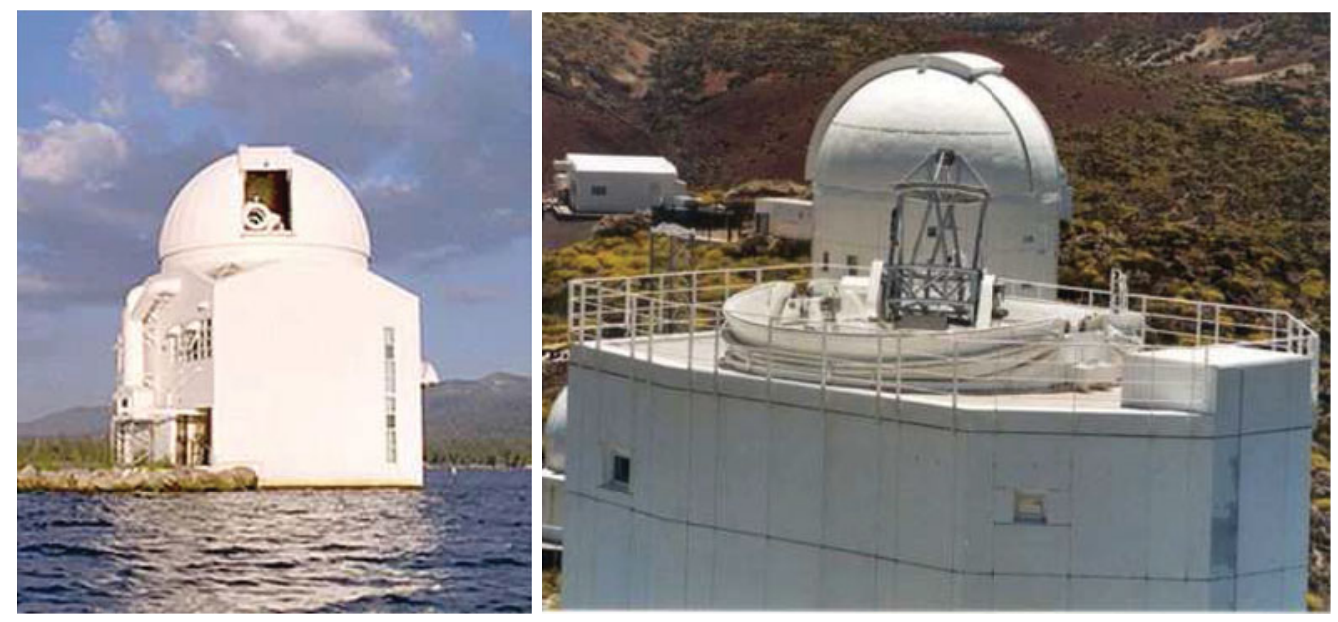

Figure 4. Left: the $1.6 \mathrm{~m}$ off-axis New Solar Telescope of the Big Bear Solar Observatory. The position on a pier within Big Bear Lake (CA) guarantees long periods of good to excellent seeing. NST has been operating since 2010. Right: the $1.5 \mathrm{~m}$, on-axis German GREGOR telescope on Tenerife, inaugurated June 2012. The unique folding dome is visible, retracted on the floor of the building.

mental step in the quest for higher apertures, and such facilities share the honor and responsibility to demonstrate the feasibility and performances of large open solar telescopes. Future facilities plan on much larger sizes, up to the impressive $4 \mathrm{~m}$ aperture of the US $A d$ vanced Technology Solar Telescope (ATST, Keil et al. (2010), Rimmele et al. (2012), Rimmele et al. (2012)), currently undergoing construction on Haleakala, Hawaii and scheduled for first light in 2018 (http://http://www.nso.edu/press/ATSTConstruction). The European Solar Telescope (EST, Collados Vera \& EST Team (2012), Socas-Navarro (2012)), currently in the design phase, will also have a $4 \mathrm{~m}$ diameter, while the ambitious Chinese Giant Solar telescope (CGST, Liu et al. (2012), whose design is currently being pursued by a consortium of Chinese Institutes with support from the Chinese Academy of Sciences foresees a giant ring telescope of a $1 \mathrm{~m}$ width with $8 \mathrm{~m}$ diameter. Such a telescope would offer many advantages in terms of low-instrumental polarization and thermal control, while still allowing a large collecting area (equivalent to a $5 \mathrm{~m}$ diameter solid mirror).

Extensive site-testing campaigns have been conducted ahead of installation of these facilities (or choice of their future site) to ensure that the best seeing characteristics are achieved over long periods of time. The ATST Team conducted a thorough survey of 6 possible sites (Socas-Navarro et al. (2005)), eventually settling on the Haleakala location due to its superior coronal characteristics, an important scientific goal of the project. Similar campaigns held in India and China respectively, have identified superb sites nearby high mountain lakes. At least two lake sites in the high altitude $(>4,000 \mathrm{~m})$ dry Ladakh desert, at the foot of the Himalaya, have been marked as possible location of the planned 2 m, India's National Large Solar Telescope (NLST, Hasan (2012)), foreseen for first light in 2017. The Fuxian Lake Solar Observatory in the Yunnan province in China has instead been chosen for the recently commissioned $1 \mathrm{~m}$ New Vacuum Solar Telescope (NVST, Ji \& Liu (2012)). The excellent seeing characteristics of the site can be appreciated in Fig. 5 which shows a speckle-reconstructed image of an active region acquired, in the $\mathrm{TiO}$ band at $706 \mathrm{~nm}$ (Ji \& Liu (2012)). 


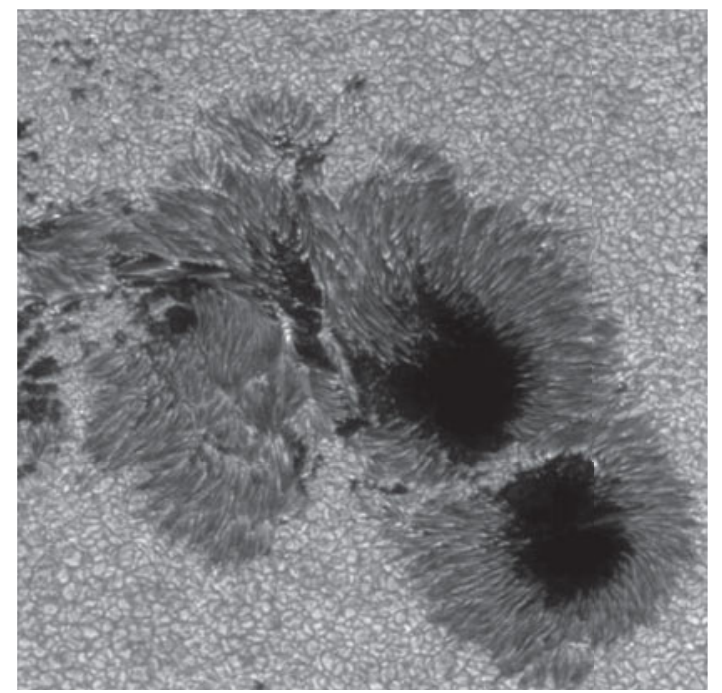

Figure 5. Active region observed in July 2011 at the NVST in the TiO band at $706 \mathrm{~nm}$. The image has been reconstructed with speckle methods from bursts of $5 \mathrm{~ms}$ exposure. From Ji \& Liu (2012).

As mentioned above, high-order AO systems are part of the original design/construction plan for all of the new ground-based telescopes, as they are necessary to achieve high Strehl ratio throughout the visible spectrum (see e.g. Richards et al. (2010), Berkefeld et al. (2012)). Still, the size of the isoplanatic patch, which determines the size of the field of view effectively diffraction limited, can be a severe limitation even in the best of conditions (e.g. Rimmele et al. (2010)). Much effort is thus currently being devoted to characterize the contributions to seeing from atmospheric layers up to 10-12 km (Scharmer \& van Werkhoven (2010), Berkefeld et al. (2010), Kellerer et al. (2012)) in order to develop and optimize the so-called Multi-Conjugate Adaptive Optics systems, which couple (multiple) deformable mirrors to a number of relevant layers in the atmosphere and allows correction on larger fields of view. These systems are currently being tested at the DST (Rimmele et al. (2010)), the GREGOR telescope (Schmidt et al. (2012)), the NST (Goode \& Cao (2012)), with the obvious scope to install them at the largest telescopes of the future (e.g. Rimmele et al. (2012), Socas-Navarro (2012)).

All the facilities described above are planned to run as multi-purpose, flexible observatories, with a large variety of scientific targets that can be addressed by versatile instrument suites (see Sect. 5 below). A somewhat different facility is the Coronal Solar Magnetism Observatory (COSMO, Tomczyk (2012), Gallagher et al. (2012)), a proposed US-China project currently completing the Preliminary Design Review. COSMO's principal goal is that of accurately, and continuously, measure the coronal field through Zeeman effect in the Fe XIII $1074.7 \mathrm{~nm}$ coronal line using the Large Coronograph, a $1.5 \mathrm{~m}$ refracting chronograph, designed to achieve a very low level of scattered light.

The balloon-borne $1 \mathrm{~m}$ telescope SUNRISE (Solanki et al. (2010)) is a unique facility, tailored for visible imaging spectra-polarimetry and near-UV imaging, taking advantage of the reduced atmospheric absorption at its operating $\approx 35 \mathrm{~km}$ of altitude. The first flight of SUNRISE in June 2009 provided a novel view of quiet-Sun magnetism at small spatial scales (Danilovic et al. (2010), see also Martinez Pillet (2012) and Sect. 4). SUNRISE is currently scheduled for a second flight in mid-2013. 


\subsection{Radio-telescopes}

Technological development in the field of radio-astronomy has recently spawned many new projects worldwide, several of which are either solar dedicated, or of general astrophysical interest but capable of observing the Sun as well.

Much like the efforts in solar optical astrophysics to obtain full spectral information coupled with spatial and temporal information, the main emphasis in contemporary solar radio-astronomy is that of developing dynamic imaging spectroscopy of the Sun in a large range of frequencies (e.g. Pick \& Vilmer (2008)). This implies the use of interferometric arrays with dynamic baseline arranging, as for example with the recent transformation of the Jansky VLA into the Expanded Very Large Array (EVLA, Perley et al. (2011)) which can currently perform, albeit sporadically, true solar imaging spectroscopy in the range 1-8 GHz (to be expanded to 1-18 GHz, see Bastian \& Gary (2012)). The same frequency range will be monitored by the solar dedicated Expanded Owens Valley Solar Array (EOVSA, Gary et al. (2011)), an array of thirteen $2.1 \mathrm{~m}$ antennas which will allow imaging spectroscopy of flares and active regions with a few arcsec spatial realution and $1 \mathrm{sec}$ temporal resolution. Completion of EOVSA is foreseen for October 2013.

The Frequency Agile Solar Radiotelescope (FASR, Bastian \& Gary (2012)) represent the next development in the US, aiming at achieving dynamic imaging spectroscopy over an extremely large frequency bandwidth, thus allowing to track phenomena in the solar atmosphere simultaneously at many heights. FASR will comprise three separate arrays of antennas sweeping the frequency range $50 \mathrm{MHz}-21 \mathrm{GHz}$ in less than $1 \mathrm{sec}$, with a max. spatial resolution of 1 arcsec. FASR, to be installed in Owens Valley, has been highly rated in the National Academies Decadal Surveys, but is currently awaiting development of a mid-scale funding line from the US National Science Foundation before it can proceed.

A similar project is that of the Chinese Solar Radioheliograph (CSRH, Yan et al. (2009)), currently under construction in a radio-quiet area of inner Mongolia, which will cover the $0.4-15 \mathrm{GHz}$ range with about 100 antennas. The first portion of the project, CSHR-I, covers the range $0.4-2 \mathrm{GHz}$ with 64 channels (40 antennas), and has been recently completed. (Li et al. (2012)). Solar observations have been obtained in early 2012 and are currently being processed for first results. The second portion of the project, CSRH-II, covering the $2-15 \mathrm{GHz}$ range with 60 antennas, is foreseen for completion at the end of 2013 (Yan et al. (2012)).

The largest recent development in radio astronomy is the Atacama Large Millimeter Array (ALMA, e.g. Busatta et al. (2012)), which employes 66, $12 \mathrm{~m}$ antennas for observations of a wide range of astrophysical sources in the $86-720 \mathrm{GHz}$ frequency range (3.4-0.3 mm). ALMA has been configured from the start to be able to observe the Sun, by using Sun-proof antennas and a set of specifically designed attenuators with potential for precise diagnostics of the quiet and active chromosphere, the low corona, as well as rapid transients such as flares (Karlický et al. (2011)). Test solar observations have been obtained with ALMA in 2011 and 2012 during the science verification phase, using an array of 13 antennas. The single-dish mosaicing procedure has been verified and calibrated, and has produced promising results in clearly identifying enhanced plage and active region emission, with spatial resolution of several arcsec (Fig. 6, Benz et al. (2012)). Interferometric observations, which will allow sub-arcsec spatial resolution at 0.3-9 mm, potentially resolving filamentary shock structures in the quiet chromosphere (e.g. Wedemeyer-Böhm et al. (2005)), are currently being tested. 


\section{ALMA $345 \mathrm{GHz}, \mathrm{AlA} / \mathrm{SDO}$ \& NoRH $17 \mathrm{GHz}$ images}
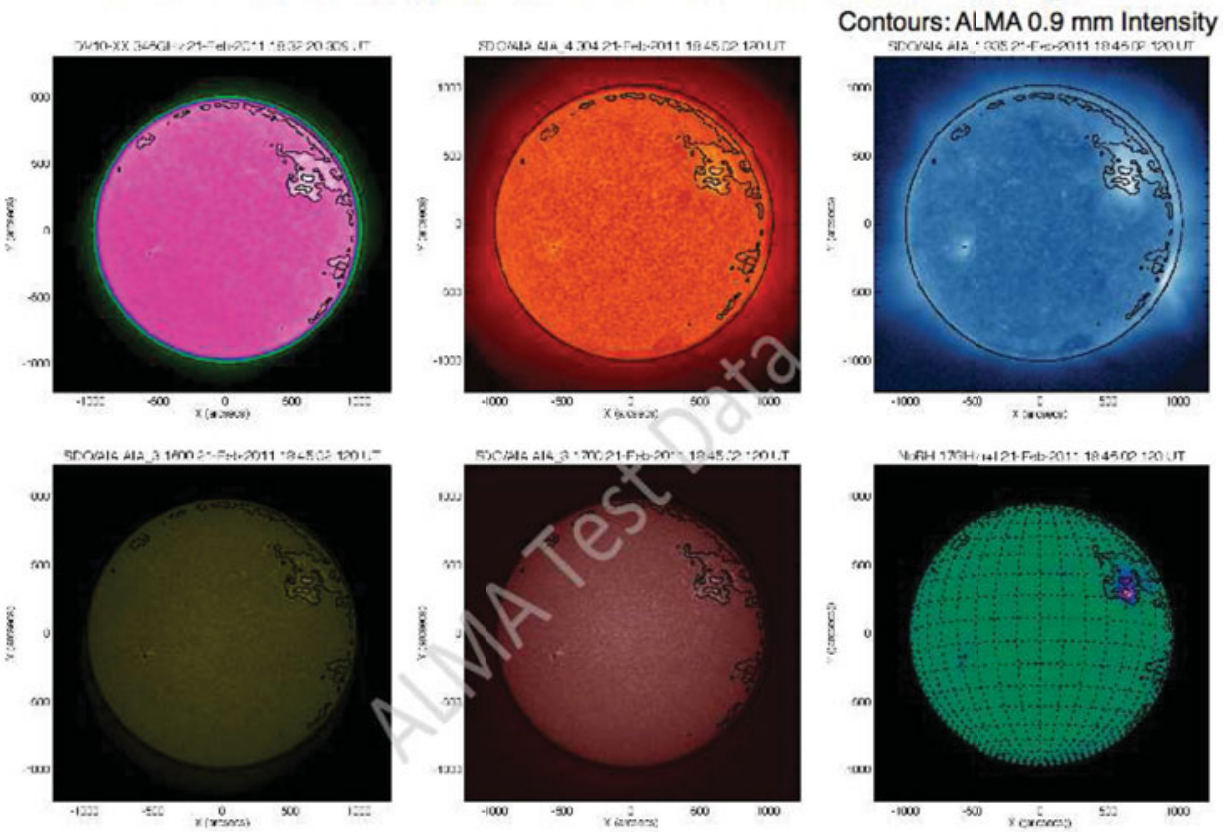

Figure 6. ALMA first solar data, from Science Verification program. From Benz et al. (2012).

\subsection{Space projects}

Several new space solar observatories are also being considered for construction in the next decade. The Chinese solar community is currently developing a project for a multipurpose solar spacecraft, the Deep-space Solar Observatory (DSO, formerly Space Solar Telescope, see Deng (2012)) which has passed budget approval in mid-Aug 2011, and whose realization will be decided upon within 2013. If successful, this will be the first scientific spacecraft launched and operated by China. The DSO will be a multi-instrument project, with emphasis on a $1 \mathrm{~m}$ optical telescope devoted to magnetic measurements in the photosphere (Fe I $532.4 \mathrm{~nm}$ ) and chromosphere (H $\beta$ line, $486.1 \mathrm{~nm})$ at $0.1-0.15$ arcsec resolution with a temporal resolution of $\sim 30 \mathrm{sec}$. The core of the magnetograph is the Two-Dimensional Real-Time Spectrograph (2DS), a multi-element birifringent filter that allows fully simultaneous observations in 8 wavelengths within a spectral line, and which is currently being prototyped (Deng (2012)). The payload includes complementary instruments as well, such as an EUV imager, an $\mathrm{H} \alpha$ and WL imager, and a Ly $\alpha$ telescope.

The Interhelioprobe solar observatory is an out-of-the-ecliptic, inner heliosphere spacecraft (perihelion $=0.28 \mathrm{AU}$ ), currently being developed within the Federal Space Program of the Russian Federation (Bogachev et al. (2012)). It is aimed at studying the Sun from close distances in the inner heliosphere, and carries a suite of 5 remote instruments (plus 15 in situ ones. These include a multi-channel EUV imager, a white-light chronograph, and a heliospheric imager, with increasingly large FOVs, to continuously couple coronal structure to inner-heliosphere conditions. Interhelioprobe is scheduled for launch in the 2018-2020 time frame, and will perform stereoscopic observations of the Sun together with Earth-based instrumentation and Solar Orbiter (Müller et al. (2012)). 
After the success of the Hinode mission, the Japanese and international communities are now proposing a next-generation mission, SOLAR-C, with a similar suite of instruments (see Shimizu et al. (2012)) but much more ambitious in scope. The goal of SOLAR-C is infact that of providing a comprehensive, seamless view of the solar atmosphere, from the photosphere to the corona, by simultaneously using instruments with complementary diagnostics capabilities (Katsukawa et al. (2012)). In particular, SOLAR-C will seek to provide diagnostics of the dynamics and magnetism of the upper chromosphere, an "interface" region which is still largely unexplored and that was missing from the Hinode payload capabilities. This will be accomplished with a large optical telescope, SUVIT, $1.5 \mathrm{~m}$ in diameter, which, coupled with the seeing-free environment of space, will rival modern ground-based instruments in terms of achieved spatial resolution. SUVIT is designed to provide spectro-polarimetric observations over a large wavelength range: again taking advantage of the lack of atmosphere, it aims at covering the chromospheric Mg II h\&k lines around $280 \mathrm{~nm}$, as well as the visible and near-infrared range up to the He I $1083 \mathrm{~nm}$ triplet (Suematsu et al. (2012)). Broad- and narrow-band filtergraphs will complement the spectral observations, although the cadence and multi-wavelength coverage achievable will depend on the actual telemetry, currently under discussion.

\section{Steps Ahead: Future Science and what the New Facilities should not Forget}

A number of state-of-the-art observational and theoretical advances are pushing current facilities to their limit, and can offer important insights and lessons for future developments. We report below some examples that were discussed during SpS6.

\subsection{Quiet Sun magnetism}

The need for high-precision polarimetry is well illustrated by the still puzzling structure of "quiet Sun" magnetic fields, as shown in Sect. 2. Given the different sensitivity of the Zeeman signal to longitudinal (LOS) or transverse fields, the typical polarimetric sensitivity of $10^{-3} I_{c}$ of modern instruments (such as the SP on Hinode, or the MTR at THEMIS, Bommier (2011)) translates into our capacity to much better discern LOS fields, with respect to the transverse ones - namely, few Gauss vs. many tens of Gauss (cf. Martinez Pillet (2012)). It is possible that such a "biased" view of solar magnetism could be the origin of some controversial results related to quiet-Sun magnetism, including the long-standing issue of why some isolated network flux patches appear to lose flux and disappear in 1-2 hours (Spruit et al. (1987)), or the existence of "flux voids" on mesogranular scales (e.g. Yelles Chaouche et al. (2011), Berrilli et al. (2013)), which would contrast with the likely exixtence of a small scale turbulent dynamo, operating at granular scales (Brandenburg (2011); see also Sect. ??).

Indeed, when pushing the data to higher sensitivity, a "sea" of field structures appears, as shown in Fig. 7 . In particular, at the $10^{-4}$ sensitivity range all flux patches are constantly interacting with opposite polarity neighbors, which might explain the reported "in-place" disappearance of unipolar flux as a sensitivity issue (Martinez Pillet (2012)). Voids instead seem to be a persistent feature; a possible resolution of the small scale dynamo at granular scale might be waiting for systematic $10^{-4} I_{c}$ observations.

A problem intrinsic to the use of Zeeman polarimetry to study weak fields is the fact that the long integration times, necessary to reach the desired polarimetric accuracy, inevitably degrade spatial resolution, leading to polarity cancellation. The complemen- 

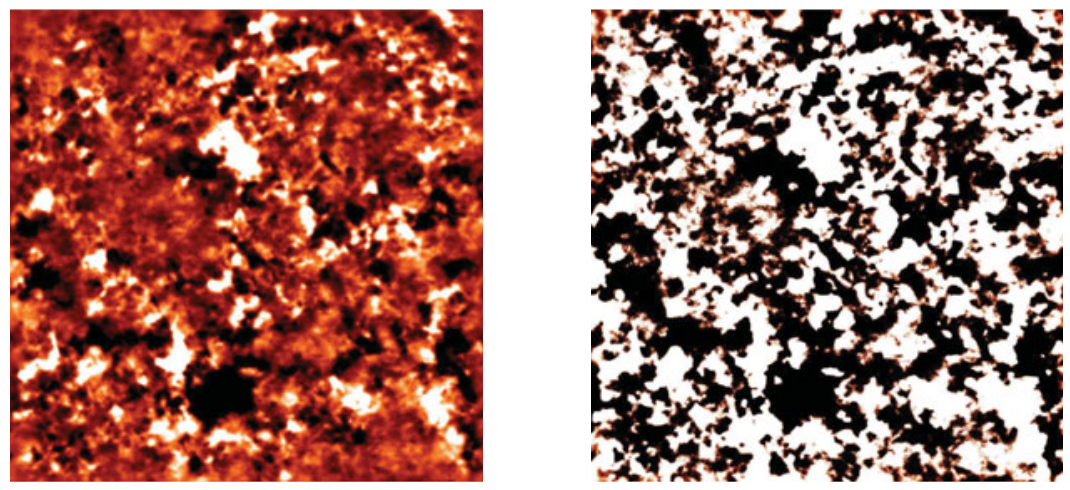

Figure 7. Left: IMaX magnetic field map of a quiet Sun region, saturated at a level of $10^{-3} I_{c}$. Right: the same map when data are pushed to $10^{-4} I_{c}$ sensitivity. From Martinez Pillet (2012).

tary use of the Hanle effect offers much promise in this respect. The Hanle effect is the modification, caused by the presence of a magnetic field, of the linear polarization produced by scattering processes in a spectral line (Belluzzi et al. (2012)). As the signal from the Hanle effect does not vanish in presence of mixed-polarities (it is proportional to $B^{2}$ ), and its sensitivity peaks between a few Gauss and $\approx 200 \mathrm{G}$, this diagnostic is well suited to investigate the magnetism of the quiet Sun (see also Faurobert (2012)). As Hanle polarimetry moved more into "mainstream" solar physics in the last years, many investigations have dealt with the presence of a weak turbulent magnetic field in the solar photosphere, whose strength has been estimated at a few Gauss up to several tens of G (e.g. Kleint et al. (2010), Faurobert et al. (2001), Bommier et al. (2005)). The use of differential Hanle diagnostics, i.e. the use of multiple lines with different sensitivity to magnetic fields (especially molecular lines such as those of $\mathrm{CN}, \mathrm{C}_{2}, \mathrm{MgH}$ ), might be able to provide more robust results to settle such controversy. Investigations of this kind have recently suggested that the turbulent field has a strong dependence with atmospheric depth (Milić \& Faurobert (2012)), as well as shown an apparent lack of relationship with solar cycle phase (e.g. Kleint et al. (2010)).

Observational difficulties related to the use of the Hanle effect lie in the weakness of the scattering linear polarization of solar lines (often a fraction of a percent); the quest for higher polarimetric sensitivity will thus much benefit the use of both Zeeman and Hanle polarimetry. It is also important to remark that many modeling assumption enter the derivation of magnetic fields once precise polarimetric measures are acquired. In particular, observed Stokes profiles are often asymmetric, indicating a variation of atmospheric parameters with height along the line of sight. This, however, has just begun to be considered in the inversion methodology using the Zeeman effect (e.g. Viticchié et al. (2011)). Analogously, the derivation of fields when using the Hanle effect relies on a number of assumptions, e.g. on the isotropy of the field, an hypothesis which has recently undergone scrutiny (e.g. Frisch et al. (2009), Faurobert (2012)).

\subsection{Polarimetry of the chromosphere and transition region}

The magnetism of the upper solar atmosphere - namely upper chromosphere and transition region, is of high scientific interest as a crucial force in shaping this complex interface between the photosphere and corona, where most of the non-thermal energy that creates the corona and solar wind is released (Judge (2009), see also Sect. 2.3). In a physical regime where most of the spectral diagnostics lie in the far-UV or EUV range, Zeeman 
polarimetry is of limited use because the (expected) small field values at these wavelengths produce a Zeeman splitting much smaller than the width of the lines. On the other hand, in the same regime a large degree of scattering polarization is expected in the spectral lines, so that the Hanle effect can be of great diagnostic potential because its magnetic sensitivity is independent of the wavelength and Doppler width of the spectral line under consideration (e.g. Trujillo Bueno (2012)).

Theoretical calculations are currently being performed to determine the degree of polarization expected in such UV spectral lines in the presence of magnetic fields, both in "classical" 1-D models of the solar atmosphere, and adopting physical parameters deriving from modern, self-consistent numeric simulations such as those presented in Leenaarts et al. (2012). Belluzzi \& Trujillo Bueno (2012) analyzed for example the behavior of the $\mathrm{Mg}$ II lines at $280 \mathrm{~nm}$, which form in the upper chromosphere, while Trujillo Bueno et al. (2012), Štěpán et al. (2012) investigated the polarization properties of the Ly $\alpha$ lines of H I and He II. These latter lines appear very good candidates for inferring fields between roughly 10 and $100 \mathrm{G}$ within the transition region; in particular, their joint use might alleviate ambiguities in the retrieval of the fields, due to, e.g., to horizontal atmospheric inhomogeneities. Still, the expected linear polarization is of the order of $1-2 \%$ at most, again highlighting the need for very precise polarimetric measurements.

As already described in Sect. 2.3, a "special" spectral line for investigation of the upper chromosphere' magnetism is the He I triplet at $1083 \mathrm{~nm}$, that lies in a completely different spectral range, namely the near-infrared. The line forms in chromospheric conditions, mostly through the photoionization-recombination mechanism (Andretta \& Jones (1997)) which implies that it is mostly visible where abundant UV radiation is present, i.e. usually in magnetic, heated structures. Its sensitivity to magnetic fields through both Hanle and Zeeman effect has been well investigated in recent years (e.g. Asensio Ramos et al. (2008)), and its use for diagnostics of fields in prominences, active regions' chromosphere, and even spicules, is rapidly increasing (Casini et al. (2009), Centeno et al. (2010), Schad et al. (2011), Schad et al. (2012), Schad et al. (2013)).

\subsection{Scattered light issues}

While image restoration has improved enormously in the last decade, affording neardiffraction limited data from ground-based telescopes (e.g. van Noort et al. (2005)), the contrast of such images is still a controversial issue. Indeed, it appears that the measured granulation contrast at several wavelengths, as measured by the SOT/Hinode (taking into account its actual Point Spread Function, derived using transit and eclipse data), it is well matched to the values deriving from modern numerical simulations (Wedemeyer-Böhm \& Rouppe van der Voort (2009)). On the contrary, the granulation contrast measured at ground-based observatories is still much lower than predicted (Uitenbroek et al. (2007), Scharmer et al. (2010)), the hypothesis being that several sources of straylight must be present, whether from the Earth's atmosphere or the combined telescope-instrumentation optical train (Löfdahl (2012)). Attempts to identify the possible sources of stray-light within a modern solar telescopes show that the fraction of scattered light in a signal can be surprisingly high, around 0.4 , and that the dominant contribution appears to derive from wavefront aberrations, which possibly originate in the deformable mirror of the adaptive optics system (Löfdahl \& Scharmer (2012)). These studies are still in their infancy, but they are paving the way for much needed investigations that will, hopefully, fully characterize the influence of scattered light and, eventually, drive our ability to correct for it, especially for what concerns chromospheric imaging and spectra. 

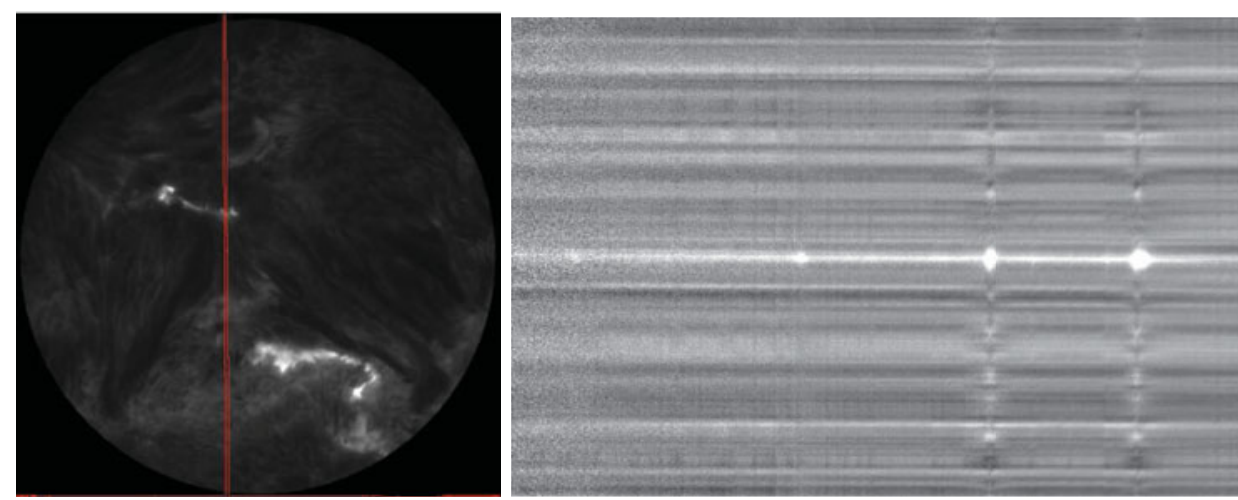

Figure 8. Left: IBIS image in $\mathrm{H} \alpha$ core during a C1.0 flare on August 18, 2011. The vertical line indicates the position of the spectrograph slit. Right: corresponding spectrum in Ca II H\&K range (over a longer slit extension than the left image). A reference spectrum has been subtracted to enhance flare signature. Easily visible is the emission form Ca II $\mathrm{H}$ and nearby $\mathrm{H} \epsilon$, Ca II K, as well as $\mathrm{H}_{8}$ and $\mathrm{H}_{9}$ pushing into the blue limit of the spectrum. The bright strip indicates (putative) continuum emission (courtesy of A. Kowalski and G. Cauzzi).

\subsection{Flare physics}

Studies of the corona during solar flares have increased ten-fold since the advent of UV and X-ray imaging and spectroscopy from satellites such as Yohkoh, SOHO, Hinode, etc. Yet, comprehensive studies of the flaring chromosphere, the main source of the flares radiative output, are still lacking. Difficulties include the complex physics involved in the rapid development of a flare within a dense medium (non-equilibrium ionization, shock and plasma waves, return currents, etc.), as well as the observationally difficulty to correctly position the instruments' FOV on chromospheric flaring kernels that are small, rapidly evolving, and occurring in mostly unpredictable locations. While this difficulty exists for coronal instruments as well, the latter usually possess a larger FOV (albeit at lower spatial resolution) and often focus on the slower decay phase of a flare, while the main value of chromospheric diagnostics is certainly in the rapid impulsive phase.

Novel developments in this field hold promise of a renewed observational and theoretical interest in the physics of chromospheric flares. One the one hand, the availability of modern radiation-hydrodynamical calculations including energy dissipation from accelerated electron beams allows a more direct link from observables to physical conditions (e.g. Allred et al. (2005), Cheng et al. (2010)). On the other hand, the advent of imaging spectrographs with large FOVs, such as IBIS, CRISP or GFPI (Cavallini (2006), Scharmer et al. (2008), Puschmann et al. (2012)) will permit a more comprehensive coverage of regions with flaring potential, while maintaining full spectral information on relevant lines (Deng et al. (2013); see also Fig. 8).

Multi-line diagnostics are also a powerful tool to identify and discriminate physical processes in flares, for example spatio-temporal details of the chromospheric heating which are ultimately related to acceleration processes in the reconnection site (Cheng et al. (2006), Kašparová \& Heinzel (2002)). Recent observations of enhancements in the infrared continuum during a particularly energetic flare (Xu et al. (2012)) have also prompted a revisit of the mechanisms that lead to the so-called white light flares (WLFs), and the recognition that broad-spectral observations at high spatial and temporal reso- 
lution are necessary for this task (e.g. Kowalski et al. (2012)). The (apparent) extreme fragmentation of the basic reconnection process, inferred in radio (Chen et al. (2013)), optical (Radziszewski et al. (2011)) and X-rays observations (Cheng et al. (2012)) highlights this need for high cadence, high spatial resolution observation while preserving spectral and spatial coverage (Deng (2012)). Large aperture telescopes appear thus necessary to reveal fine flaring structures at $0.1 \mathrm{arcsec}$ and $0.01 \mathrm{sec}$ resolution, to reveal the nature of WLFs and elementary bursts.

\section{Impact of new Large Facilities on Key Scientific Questions}

The scientific issues discussed in Sect. 2 and 4 clearly outline many requirements that new facilities will have to comply with, in order to properly address relevant science.

Routinely achieving a high polarimetric sensitivity, of the order of $10^{-4} I_{c}$ or better, as necessary for weak field studies and Hanle polarimetry, will require telescopes with apertures sensibly larger than what currently available. The $1.5 \mathrm{~m}$ class NST and GREGOR telescopes are just starting to tackle the issue of measuring polarization with dedicated instrumentation (Cao et al. (2010), Hofmann et al. (2012)), and will in the coming years reveal fascinating science as well as (probable) technical limitations. However, it is widely acknowledged that a quantum leap in this field will occur when the planned 4-m class telescopes will come online later in the decade, with their design for highest throughput (through all reflective optics, and minimal number of surfaces). Yet, one has to constantly remember that any telescope, when working at the diffraction limit, will collect the same number of photons no matter the aperture (see Martinez Pillet (2012))! Worse still, to maintain a higher spatial resolution, exposure times will need to be shorter - effectively reducing the signal-to-noise ratio of data acquired with larger facilities. This poses an interesting conundrum, whereas to provide the much needed improvement in polarimetric accuracy, one will have to purposely limit the spatial resolution to be achieved with large telescopes, using them more like "light bucket" rather than high resolution facilities. This is the approach taken for example for some of the polarimetric instruments planned for the ATST, like the near- to mid-infrared CryoNIRSP (optimized for coronal physics, see Kuhn et al. (2012)).

A second, important problem in the quest for high polarimetric accuracy will be the ability to characterize and control telescope and instrumental polarization (as already remarked more than 30 years ago! by Stenflo (1982)). Large telescopes are adopting different strategies to address this issue. Both GREGOR and the future EST will use on-axis designs to minimize polarization from the main optical elements. The preliminary design of EST is pushing further this concept by developing the optical path, all the way to the Coude' focus, with a 14-mirror "compensated" design so to minimize the instrumental polarization induced by the telescope and make it independent of the solar elevation and azimuth, at once (Collados Vera \& EST Team (2012), Socas-Navarro (2012)). The NST and ATST have instead an off-axis design, chosen to optimize telescope PSF and minimize scattered light, with the latter property being especially relevant for use of ATST for coronal physics (Rimmele et al. (2012), see below). Recent investigations, using both theoretical models and actual solar data, indicate that techniques recently adopted to precisely infer polarization properties of the main optical elements of telescopes like the DST will be applicable to the ATST to achieve the required precision (Elmore (2012)). 
Coronal polarimetry will push even further telescope requirements, due to the combination of intrinsically weak magnetic signals and brightness of the background with respect to the actual coronal intensity. Thus modern projects are striving to provide, together with larger apertures, very low levels of scattered light deriving from the main optics. To reach this goal, ATST, which has coronal physics among its main scientific objectives, adopted an off-axis design, strict figures for the main mirror, as well as procedures for contamination control of the primary and secondary mirrors (McMullin et al. (2012)). The much reduced scattered light will also benefit accurate measures of the physical properties of small structures in, for example, sunspots. The COSMO project is instead adopting a unique solution, i.e. a $1.5 \mathrm{~m}$ refractive telescope, as lenses provide better scattered light levels and control with respect to a mirror (Tomczyk (2012)).

The majority of the new and planned facilities are designed to function as full observatories, i.e. with multiple and flexible instrumentation that can address a variety of scientific issues. Indeed, the emphasis of many new facilities is the simultaneous use of varied instrumentation that can observe at different wavelengths and/or with different capabilities. In particular, the combined use of "classical" slit-spectrographs and imagers, either broadband, or tunable narrow-band ones, appears as the most promising approach (see e.g. Fig. 8, or Judge et al. (2010), using the DST imaging spectroscopy together with UV and IR spectroscopy), and is foreseen as the standard observing mode of large facilities (see e.g. Fig. 9).
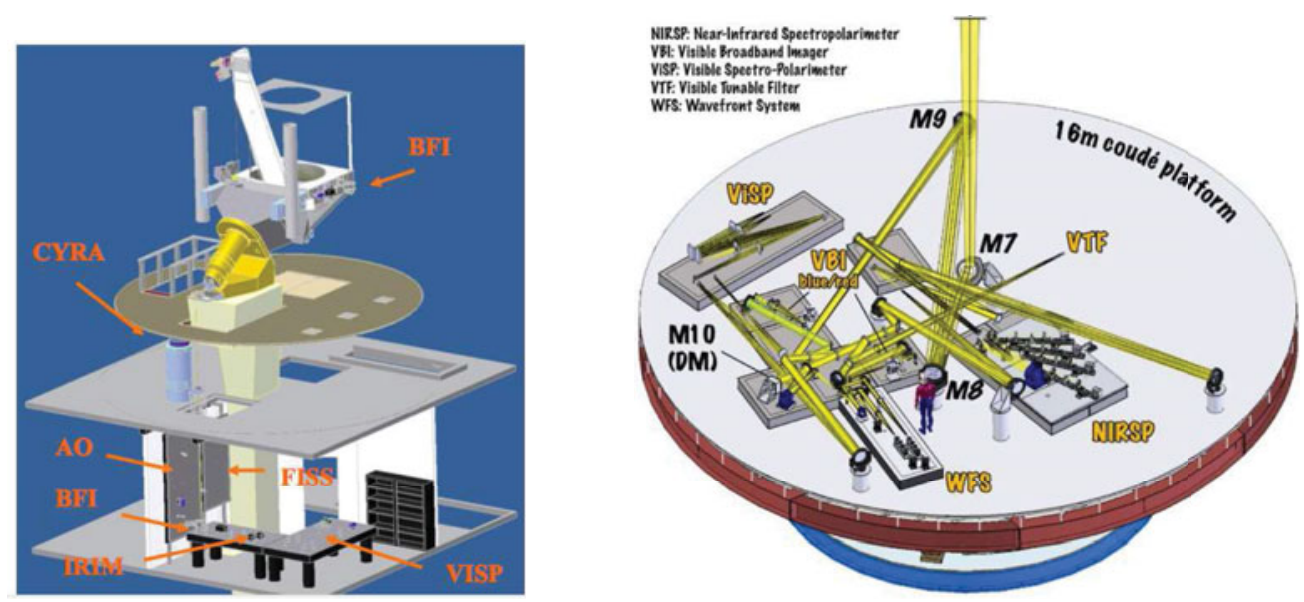

Figure 9. Schematic representation of the NST (left) and ATST (right) optical arrangement, showing capability to observe simultaneously with multiple instruments.

The large wavelength coverage is also a defining characteristics of most new telescopes. ATST will be able to operate from $350 \mathrm{~nm}$ up to thermal infrared (Rimmele et al. (2012)), exploiting, for example, the far-blue range for molecular spectroscopy (see previous Section), and the far-red range for coronal spectro-polarimetry (see Kuhn et al. (2012)). It is interesting to note that many of the new telescopes will enjoy diffraction-limited conditions at near-infrared wavelengths for very large fractions of the observing time (up to $80 \%$ for Haleakala! see Socas-Navarro et al. (2005))). It is thus expected that much emphasis will be give to (near) IR observations in the coming years. 


\section{Lesson Learned, and Future Directions for Telescope Operation}

\subsection{Observing strategies for future solar facilities}

A very advantageous characteristics of ground-based solar telescopes devoted to highresolution observations, has always been the large flexibility offered in instrumental configurations and observing programs. This permits the acquisition of unique observations easily adapted to ever-evolving targets and scientific questions (e.g. Uitenbroek (2006)). Traditionally, ground based telescopes have nurtured this flexibility by assigning fixed observing periods (of one to two weeks duration) to Principal Investigators traveling to the facility, during which the available instrumentation would be suited to the scientific goals of the peculiar observing program.

Such extreme flexibility can result however also in large inefficiencies of the system. First of all, the time necessary to modify the instrumental setup, sometimes substantially, accounts for a large fraction of the available observing time, of the order of $20 \%$ or more. Second, the fixed assigned dates might not optimally match with the desired observing target, whether an active region, an eruptive event, or specific values of sky transparency and turbulence levels ("seeing"), thus significantly reducing the observing duty cycle. Last, but not least, the "uniqueness" of each observing program makes it difficult to devise and utilize standard data reduction pipelines, forcing PIs to spend a large amount of time before obtaining science-ready data, and making it difficult to share data between scientists. Indeed, data acquired at modern (ground based) solar telescopes are usually proprietary, and very little of it gets ever used for more than one particular scientific objective, resulting in a generally modest record of publications/year for any given telescope (e.g. Reardon et al. (2009)). This is in stark contrast with the public-data policy adopted by most solar space observatories, which has resulted in wide-spread use and considerable scientific productivity (see e.g. the list of publications by Hinode, at http://hinode.nao.ac.jp/publ/hsc_paper_e_main.shtml).

For all these reasons, a discussion about different operational modes for future large ground-based solar facilities has been initiated in the community, with the long-term goal of enhancing their efficiency and scientific output to a level commensurable with their high cost of construction and operation. Much discussion has then been given in SpS6 to the so-called "service mode", where a prioritized list of observing programs is dynamically scheduled and executed by the telescope staff (resident astronomers and operators), optimizing the match between scientific requirements and instrumental, target, and atmospheric conditions (e.g. Silva (2001)). Service mode operations represent a significant fraction of the observing time at several night-time facilities, such as the VLT or Gemini; beside being very favorably judged by users, it has resulted in significantly improving the scientific output of these facilities (Comerón et al. (2006), Puxley \& Jørgensen (2006)). The recently commissioned ALMA radio telescope has been built to operate exclusively in this mode (Andreani \& Zwaan (2008)).

It must be noted however that a number of requirements must be satisfied for service mode operations to be highly effective, including a clear communications with the community about users' expectations vs. the real capabilities of the telescope and instrumentation, clear rules to allow the resident astronomers to undertake decisions. In particular, the VLT experience has shown the necessity of a software infrastructure for supporting the end-to-end operations of the facility, including tools for the preparation, scheduling and execution of observations, as well as archiving of data, pipeline data reduction and quality control (Peron (2012)). 
ATST is expected to operate predominantly in service mode (Uitenbroek \& Tritschler (2012), Berger (2012)). Many topics still need to be addressed and flushed-out, including the handling of "guaranteed time" for partner institutions, the communication and relationship in real time with a remote PI, and in general the design and implementation of a robust software infrastructure like that described above (Berger (2012)). However, the intervening time between now and actual ATST operation can be used to solve many of these issues and gain practical skills. Much can certainly be learned by analyzing the experience of night-time facilities, but the special circumstances of solar observations require also some "hands-on" experience at existing telescopes. After an early attempt at optimize coordinate DST observations with Hinode (Reardon et al. (2009)), the NSO has nowdevised a "service mode experiment" in preparation for ATST operations to explicitly start confronting the problems that might be encountered with this operational mode (Uitenbroek \& Tritschler (2012)). This experiment has been planned to run for one month at the beginning of 2013, utilizing a selected subset of instruments at the DST, including IBIS, ROSA and FIRS. The availability of stable, well calibrated instrumentation that can be used in relatively standard configurations is an essential pre-requisite for service mode operations, as it allows for an easier planning of observations, the sharing of calibration between different observing programs, as well as the rapid switches required by the optimization of target vs. sky conditions. $\dagger$

Another important aspect of successful service mode operations is the availability of standardized, robust data reduction pipelines which can minimize the effort needed by the PIs in order to obtain science-ready data. This has long been a problem in high-resolution solar data: on the one hand, both the ever-changing instrumental configurations and the vagaries of the seeing conspire against the development of stable and well distributed data pipelines; on the other, the extreme specialization required to analyze each and every high-resolution dataset has prevented the involvement of a larger user-base, with detriment to the scientific productivity of the field (Reardon et al. (2009)). Several groups are currently trying to overcome this limitation. In particular, the recently funded EU project SOLARNET (http://www.iac.es/proyecto/solarnet/) is planning a Europe-wide effort to increase impact of high-resolution data by offering science-ready data and facilitate their usage throughout a larger fraction of the solar community. A major part of this goal is the coordinate development of largely automatic data-reduction pipelines and archival data access for a wide variety of high resolution solar instrumentation (Uitenbroek \& Tritschler (2012)).

\subsection{Access to large databases: the lessons from SDO}

At its best, service mode operation is foreseen to increase scientific output of future telescopes by optimizing the acquisition of data with respect to local and solar conditions, targets of opportunity, etc. A "collateral effect" of such an efficient data acquisition scheme, however, is that the future large telescopes will produce enormous data volumes, of the order of tens to hundreds of TB per day (e.g. Ermolli et al. (2012)). Such volumes are not yet commonly managed in current telescopes: although some instruments come close to achieving those lower limits, (see e.g. Jess et al. (2010)), they are not operated

$\dagger$ Note: The DST service mode experiment has been run from Jan 15 through Feb 15, 2013, and has attracted considerable attention from the community, with a total of 21 requests for observing programs. About half of them have been executed during the 30-day period (most of the remaining programs have not been executed due to weather conditions and lack of suitable targets). This can be contrasted with the typical number of programs run in a comparable period in "classical" mode, which is in the range of 3-4. 
daily! A large effort in the development of viable large telescopes will then have to be spent in the intervening years before their actual operation in planning for their data storage, reduction, and analysis.

Probably the most appropriate comparison with existing facilities, in terms of data handling, is with the Solar Dynamics Observatory (Pesnell et al. (2012)). The SDO has been operating since spring of 2010, and archives daily about 1.5-2 TB (a factor of 3000 larger than what SOHO did!, Schrijver (2012)), for a projected total of 2 PB over the mission's lifetime. While virtually all of this database is freely accessible by the community, its sheer volume requires a different approach than to "normal" data. On the one hand, it is impossible for any scientist to just browse through all of the data to identify interesting events; on the other, it would be a waste of the enormous scientific potential of the SDO database if only a small fraction of it would ever be utilized because of limited manpower. Hence, barring a sensible increase of the budget devoted to data analysis (unlikely), the community needs an array of tools to optimize scientific efficiency to properly exploit this data bounty.

A large effort by the SDO Team has thus gone into optimizing the process of archive searches and data distribution, for example by distributing focused, smaller amounts of data to relevant institutes, or keeping tracks of data requests, maintaing the most popular data sets available for immediate export (Schrijver (2012)). Earlier missions like TRACE have paved the way for the use of standard software like SolarSoft (http://www.lmsal. com/solarsoft, originally used for Yohkoh data) and standard searches through the Virtual Solar Observatory (http://vso.nso.edu), based on parameters' search such as instrument, temporal intervals etc. While the Hinode mission pushed archival searches to the more complex case of high volume, spectroscopic imaging instruments and their flexible use (http://sdc.uio.no), the SDO Team has further expanded on these capabilities by introducing an "event search system" (iSolSearch, http://www.lmsal.com/isolsearch) that enables searching on data contents rather than instrument attributes and settings. The search is based on a list of "event classes", such as flares, filaments, active regions, etc, to which new events can constantly be added to be "recognized" by the system.

Similar efforts will have to be carried out for databases of future large facilities, with the ultimate goal of a fully-shared approach between archival of space missions and ground-based telescopes' data (Reardon (2012)). Many of the lessons learned from SDO can be incorporated in the design and implementation of such systems, including the need of involving actual users in the development of archives and data-retrieving tools, and adopting a design open to modifications and system changes to accommodate evolving technology and user needs (Schrijver (2012)).

\section{Conclusions}

Large solar telescopes hold promise of revolutionizing our knowledge of the solar atmosphere and its dynamics, but pose formidable challenges for what concern their full implementation, and future operation. The scale of these challenges is such that the solar community needs to gather all forces to be able to properly address technical and scientific hurdles, from the handling of telescope polarization to the development of proper interpretational tools to address observed new phenomena.

Special Session 6 exploited the interdisciplinary and international character of the IAU to start gather interested and involved scientists, and discuss some of the more pressing issues in this field. The enthusiastic response of the community made clear that such 
a shared effort is high in the list of priorities, and made for a very lively and fruitful discussion, which will continue in the years ahead. In particular, we expect that the next IAU General Assembly in Honolulu in 2015 will be a proper venue to host a sequel of this discussion, also considering the ongoing construction of ATST in the Hawaiian island of Maui.

\section{Acknowledgements}

Travel funds for several participants were provided by grants No. 11178005 and 10921303 of the National Natural Science Foundation of China, No. 2011CB811401 of the Ministry of Science and Technology of China, and No. KJCX2-EW-T07 of the Chinese Academy of Sciences, as well as from the Metcalf Travel Award of the US Solar Physics Division. We gratefully acknowledge their support.

The National Solar Observatory is operated under a cooperative agreement between the Association of Universities for Research in Astronomy, Inc. (AURA), and the National Science Foundation.

\section{References}

Allred, J. C., Hawley, S. L., Abbett, W. P., \& Carlsson, M. 2005, ApJ, 630, 573

Andreani, P. \& Zwaan, M. 2008, vol. 7016, SPIE Conf. Ser.

Andretta, V. \& Jones, H. P. 1997, ApJ, 489, 375

Asensio Ramos, A. 2009, ApJ, 701, 1032

Asensio Ramos, A., Trujillo Bueno, J., \& Landi Degl'Innocenti, E. 2008, ApJ, 683, 542

Attie, R., Innes, D. E., \& Potts, H. E. 2009, A\&A (Letters), 493, L13

Balmaceda, L., Vargas Domínguez, S., Palacios, J., Cabello, I., \& Domingo, V. 2010, A\&A (Letters), 513, L6

Bastian, T. \& Gary, D. 2012, IAU Special Session, 6, E2.15

Beck, C. \& Rezaei, R. 2009, A\&A, 502, 969

Beeck, B., Collet, R., Steffen, M., Asplund, M., Cameron, R. H., Freytag, B., Hayek, W., Ludwig, H.-G., \& Schüssler, M. 2012, A\&A, 539, A121

Belluzzi, L. \& Trujillo Bueno, J. 2012, ApJ (Letters), 750, L11

Belluzzi, L., Trujillo Bueno, J., \& Štěpán, J. 2012, ApJ (Letters), 755, L2

Benz, A. O., Brasja, R., Shimojo, M., Karlicky, M., \& Testi, L. 2012, IAU Special Session, 6, $\mathrm{E} 2.05$

Berger, T. 2012, IAU Special Session, 6, E2.01

Berkefeld, T., Schmidt, D., Soltau, D., von der Lühe, O., \& Heidecke, F. 2012, Astron. Nachr., 333,863

Berkefeld, T., Bettonvil, F., Collados, M., López, R., Martín, Y., Peñate, J., Pérez, A., Scharmer, G. B., Sliepen, G., Soltau, D., Waldmann, T. A., \& van Werkhoven, T. 2010, vol. 7733, SPIE Conf. Ser.

Berrilli, F., Scardigli, S., \& Giordano, S. 2013, Solar Phys., 282, 379

Bogachev, S., Kuzin, S., Shestov, S., \& Perzov, A. 2012, IAU Special Session, 6, E2

Bommier, V. 2011, A\&A, 530, A51

Bommier, V., Derouich, M., Landi Degl'Innocenti, E., Molodij, G., \& Sahal-Bréchot, S. 2005, $A \mathscr{G} A, 432,295$

Bommier, V., Martínez González, M., Bianda, M., Frisch, H., Asensio Ramos, A., Gelly, B., \& Landi Degl'Innocenti, E. 2009, A $\& A$ A, 506, 1415

Bonet, J. A., Márquez, I., Sánchez Almeida, J., Cabello, I., \& Domingo, V. 2008, ApJ (Letters), 687, L131

Bonet, J. A., Márquez, I., Sánchez Almeida, J., Palacios, J., Martínez Pillet, V., Solanki, S. K., del Toro Iniesta, J. C., Domingo, V., Berkefeld, T., Schmidt, W., Gandorfer, A., Barthol, P., \& Knölker, M. 2010, ApJ (Letters), 723, L139

Brandenburg, A. 2011, ApJ, 741, 92 
Brandt, P. N., Scharmer, G. B., Ferguson, S., Shine, R. A., \& Tarbell, T. D. 1988, Nature, 335, 238

Busatta, A., Brunelli, A., Rampini, F., \& Marchiori, G. 2012, vol. 8450, SPIE Conf. Ser.

Cao, W. 2012, IAU Special Session, 6, E2.02

Cao, W., Gorceix, N., Coulter, R., Ahn, K., Rimmele, T. R., \& Goode, P. R. 2010, Astron. Nachr., 331, 636

Casini, R., Manso Sainz, R., \& Low, B. C. 2009, ApJ (Letters), 701, L43

Cattaneo, F. 1999, ApJ (Letters), 515, L39

Cavallini, F. 2006, Solar Phys., 236, 415

Centeno, R., Socas-Navarro, H., Lites, B., Kubo, M., Frank, Z., Shine, R., Tarbell, T., Title, A., Ichimoto, K., Tsuneta, S., Katsukawa, Y., Suematsu, Y., Shimizu, T., \& Nagata, S. 2007, ApJ (Letters), 666, L137

Centeno, R., Trujillo Bueno, J., \& Asensio Ramos, A. 2010, ApJ, 708, 1579

Chen, B., Bastian, T. S., White, S. M., Gary, D. E., Perley, R., Rupen, M., \& Carlson, B. 2013, ApJ (Letters), 763, L21

Cheng, J. X., Qiu, J., Ding, M. D., \& Wang, H. 2012, A\& A, 547, A73

Cheng, J. X., Ding, M. D., \& Carlsson, M. 2010, ApJ, 711, 185

Cheng, J. X., Ding, M. D., \& Li, J. P. 2006, ApJ, 653, 733

Collados Vera, M., EST Team 2012, ASP Conf. Ser., 463, 413

Comerón, F., Mathys, G., Kaufer, A., Hainaut, O., Hanuschik, R., Romaniello, M., Silva, D., \& Quinn, P. 2006, vol. 6270, SPIE Conf. Ser.

Danilovic, S., Beeck, B., Pietarila, A., Schüssler, M., Solanki, S. K., Martínez Pillet, V., Bonet, J. A., del Toro Iniesta, J. C., Domingo, V., Barthol, P., Berkefeld, T., Gandorfer, A., Knölker, M., Schmidt, W., \& Title, A. M. 2010, ApJ (Letters), 723, L149

Danilovic, S., Schüssler, M., \& Solanki, S. K. 2010, A\&广A, 513, A1

de la Cruz Rodríguez, J., De Pontieu, B., Carlsson, M., \& Rouppe van der Voort, L. H. M. 2013, ApJ (Letters), 764, L11

Deng, N., Tritschler, A., Jing, J., Chen, X., Liu, C., Reardon, K., Denker, C., Xu, Y., \& Wang, H. 2013, arXiv1304.4171

Deng, Y. 2012, IAU Special Session, 6, E2.09

Denker, C., Lagg, A., Puschmann, K. G., Schmidt, D., Schmidt, W., Sobotka, M., Soltau, D., Strassmeier, K. G., Volkmer, R., von der Lühe, O., Solanki, S. K., Balthasar, H., Bello Gonzalez, N., Berkefeld, T., Collados Vera, M., Hofmann, A., \& Kneer, F. 2012, IAU Special Session, 6, E2.03

De Pontieu, B., McIntosh, S. W., Carlsson, M., et al. 2011, Science, 331, 55

De Pontieu, B., McIntosh, S. W., Hansteen, V. H., \& Schrijver, C. J. 2009, Apj (Letters), 701, L1

De Pontieu, B., Hansteen, V. H., Rouppe van der Voort, L., van Noort, M., \& Carlsson, M. 2007, ApJ, 655, 624

de Pontieu, B., McIntosh, S., Hansteen, V. H., et al. 2007, PASJ, 59, 655

De Rosa, M. L., Schrijver, C. J., Barnes, G., et al. 2009, Apj, 696, 1780

Domingo, V., Fleck, B., \& Poland, A. I. 1995, Solar Phys., 162, 1

Elmore, D. F. 2012, ASP Conf. Ser., 463, 307

Ermolli, I., Cauzzi, G., Collados, M., Paletou, F., Reardon, K., Aboudarham, J., Cirami, R., Cosentino, R., Del Moro, D., Di Marcantonio, P., Giorgi, F., Lafon, M., Pietropaolo, E., \& Romano, P. 2012, vol. 8448, SPIE Conf. Ser.

Fang, F., Manchester, W., Abbett, W. P., \& van der Holst, B. 2010, ApJ, 714, 1649

Faurobert, M. 2012, IAU Special Session, 6, E3.01

Faurobert, M., Arnaud, J., Vigneau, J., \& Frisch, H. 2001, A\&A, 378, 627

Freytag, B., Steffen, M., Ludwig, H.-G., Wedemeyer-Böhm, S., Schaffenberger, W., \& Steiner, O. 2012, Journal of Computational Physics, 231, 919

Frisch, H., Anusha, L. S., Sampoorna, M., \& Nagendra, K. N. 2009, A\& A, 501, 335

Gallagher, D., Tomczyk, S., Zhang, H., \& Nelson, P. G. 2012, vol. 8444, SPIE Conf. Ser.

Gary, D. E., Hurford, G. J., Nita, G. M., White, S. M., Tun, S. D., Fleishman, G. D., \& McTiernan, J. M. 2011, AAS/Solar Physics Division Abstracts \#42, 102

Georgobiani, D., Stein, R., \& Nordlund, A. 2012, IAU Special Session, 6, E1.02 
Gömöry, P., Beck, C., Balthasar, H., et al. 2010, A\&A, 511, A14

Goode, P. R. \& Cao, W. 2012, vol. 8444, SPIE Conf. Ser.

Gudiksen, B. V., Carlsson, M., Hansteen, V. H., et al. 2011, A\&A, 531, A154

Hagenaar, H. J., Schrijver, C. J., \& Title, A. M. 2003, ApJ, 584, 1107

Harvey, J. W., Branston, D., Henney, C. J., \& Keller, C. U., SOLIS and GONG Teams 2007, ApJ (Letters), 659, L177

Hasan, S. S. 2012, IAU Special Session, 6, E2.11

Heggland, L., De Pontieu, B., \& Hansteen, V. H. 2007, ApJ, 666, 1277

Hofmann, A., Arlt, K., Balthasar, H., Bauer, S. M., Bittner, W., Paschke, J., Popow, E., Rendtel, J., Soltau, D., \& Waldmann, T. 2012, Astron. Nachr., 333, 854

Ishikawa, R. 2012, IAU Special Session, 6, E1.03

Ishikawa, R. \& Tsuneta, S. 2010, ApJ (Letters), 718, L171

Ishikawa, R. \& Tsuneta, S. 2011, ApJ, 735, 74

Ishikawa, R., Tsuneta, S., \& Jurčák, J. 2010, ApJ, 713, 1310

Jess, D. B., Mathioudakis, M., Christian, D. J., Keenan, F. P., Ryans, R. S. I., \& Crockett, P. J. 2010, Solar Phys., 261, 363

Ji, H. \& Liu, Z. 2012, IAU Special Session, 6, E2.04

Judge, P. \& Casini, R. 2012, IAU Special Session, 6, E1.06

Judge, P. G., Reardon, K., \& Cauzzi, G. 2012, ApJ (Letters), 755, L11

Judge, P. G., Tritschler, A., \& Chye Low, B. 2011, ApJ (Letters), 730, L4

Judge, P. G. 2009, ASP Conf. Ser., 415, 7

Judge, P. G., Centeno, R., Tritschler, A., Uitenbroek, H., Jaeggli, S., \& Lin, H. 2010, AGU Fall Meeting Abstracts, A1783

Karlický, M., Bárta, M., Dąbrowski, B. P., \& Heinzel, P. 2011, Solar Phys., 268, 165

Kato, Y., Steiner, O., Steffen, M., \& Suematsu, Y. 2011, ApJ (Letters), 730, L24

Katsukawa, Y., Watanabe, T., Hara, H., Ichimoto, K., Kubo, M., Kusano, K., Sakao, T., Shimizu, T., Suematsu, Y., \& Tsuneta, S. 2012, IAU Special Session, 6, E2.07

Kašparová, J. \& Heinzel, P. 2002, A\&A, 382, 688

Keil, S. L., Rimmele, T. R., \& Wagner, J., ATST Team 2010, Astron. Nachr., 331, 609

Kellerer, A., Gorceix, N., Marino, J., Cao, W., \& Goode, P. R. 2012, A\&\&A, 542, A2

Khomenko, E. V., Collados, M., Solanki, S. K., Lagg, A., \& Trujillo Bueno, J. 2003, A\&A, 408, 1115

Kitiashvili, I., Abramenko, V., Goode, P. R., Kosovichev, A., Mansour, N., Wray, A., \& Yurchyshyn, V. 2012, IAU Special Session, 6, E1.04

Kitiashvili, I. N., Kosovichev, A. G., Mansour, N. N., \& Wray, A. A. 2011, ApJ (Letters), 727, L50

Kitiashvili, I. N., Kosovichev, A. G., Mansour, N. N., \& Wray, A. A. 2012, ApJ (Letters), 751, L21

Kleint, L., Berdyugina, S. V., Shapiro, A. I., \& Bianda, M. 2010, A\&3A, 524, A37

Klimchuk, J. 2012, IAU Special Session, 6, E1.07

Klimchuk, J. A. 2012, Journal of Geophysical Research (Space Physics), 117, 12102

Kobel, P., Solanki, S. K., \& Borrero, J. M. 2011, A\&A, 531, A112

Khomenko, E. \& Collados, M. 2012, ApJ, 747, 87

Kosugi, T., Matsuzaki, K., Sakao, T., Shimizu, T., Sone, Y., Tachikawa, S., Hashimoto, T., Minesugi, K., Ohnishi, A., Yamada, T., Tsuneta, S., Hara, H., Ichimoto, K., Suematsu, Y., Shimojo, M., Watanabe, T., Shimada, S., Davis, J. M., Hill, L. D., Owens, J. K., Title, A. M., Culhane, J. L., Harra, L. K., Doschek, G. A., \& Golub, L. 2007, Solar Phys., 243, 3

Kowalski, A. F., Hawley, S. L., Holtzman, J. A., Wisniewski, J. P., \& Hilton, E. J. 2012, Solar Phys., 277, 21

Kuhn, J. R., Scholl, I. F., \& Mickey, D. L. 2012, ASP Conf. Ser., 463, 207

Leake, J. E. \& Linton, M. G. 2013, ApJ, 764, 54

Leenaarts, J., Pereira, T., \& Uitenbroek, H. 2012, A\& $A$, 543, A109

Leenaarts, J., Carlsson, M., Hansteen, V., \& Rutten, R. J. 2007, A\&A, 473, 625

Lemen, J. R., Title, A. M., Akin, D. J., et al. 2012, Solar Phys., 275, 17

Li, S., Yan, Y., Wang, W., \& Liu, D. 2012, IAU Special Session, 6, E5 
Lites, B., Socas-Navarro, H., Kubo, M., Berger, T., Frank, Z., Shine, R. A., Tarbell, T. D., Title, A. M., Ichimoto, K., Katsukawa, Y., Tsuneta, S., Suematsu, Y., \& Shimizu, T. 2007, PASJ, 59,571

Lites, B. W., Elmore, D. F., \& Streander, K. V. 2001, ASP Conf. Ser., 236, 33

Lites, B. W., Kubo, M., Socas-Navarro, H., Berger, T., Frank, Z., Shine, R., Tarbell, T., Title, A., Ichimoto, K., Katsukawa, Y., Tsuneta, S., Suematsu, Y., Shimizu, T., \& Nagata, S. 2008, ApJ, 672, 1237

Liu, Z., Deng, Y., \& Ji, H. 2012, IAU Special Session, 6, E2

Livingston, W. \& Harvey, J. 1971, IAU Symposium, 43, 51

Löfdahl, M. 2012, IAU Special Session, 6, E3.05

Löfdahl, M. G. \& Scharmer, G. B. 2012, A\& $A, 537$, A80

Martínez González, M. J., Asensio Ramos, A., López Ariste, A., \& Manso Sainz, R. 2008, Aש̇A, 479,229

Martínez González, M. J., Manso Sainz, R., Asensio Ramos, A., \& Bellot Rubio, L. R. 2010, $A p J$ (Letters), 714, L94

Martinez Pillet, V. 2012, IAU Special Session, 6, E3.04

Martinez-Sykora, J. 2012, IAU Special Session, 6, E1.05

Martínez-Sykora, J., De Pontieu, B., \& Hansteen, V. 2012, ApJ, 753, 161

Martínez-Sykora, J., Hansteen, V., De Pontieu, B., \& Carlsson, M. 2009, ApJ, 701, 1569

McMullin, J. P., Rimmele, T. R., Keil, S. L., Warner, M., Barden, S., Bulau, S., Craig, S., Goodrich, B., Hansen, E., Hegwer, S., Hubbard, R., McBride, W., Shimko, S., Wöger, F., \& Ditsler, J. 2012, vol. 8444, SPIE Conf. Ser.

Milić, I. \& Faurobert, M. 2012, A $\& A, 547$, A38

Moll, R., Cameron, R. H., \& Schüssler, M. 2011, A\&A, 533, A126

Müller, D., Marsden, R. G., St. Cyr, O. C., \& Gilbert, H. R. 2012, Solar Phys.

Nordlund, Å. 1985, Solar Phys., 100, 209

Orozco Suárez, D., Bellot Rubio, L. R., Del Toro Iniesta, J. C., Tsuneta, S., Lites, B., Ichimoto, K., Katsukawa, Y., Nagata, S., Shimizu, T., Shine, R. A., Suematsu, Y., Tarbell, T. D., \& Title, A. M. 2007, PASJ, 59, 837

Orozco Suárez, D., Bellot Rubio, L. R., del Toro Iniesta, J. C., Tsuneta, S., Lites, B. W., Ichimoto, K., Katsukawa, Y., Nagata, S., Shimizu, T., Shine, R. A., Suematsu, Y., Tarbell, T. D., \& Title, A. M. 2007, ApJ (Letters), 670, L61

Pereira, T. M. D., De Pontieu, B., \& Carlsson, M. 2012, ApJ, 759, 18

Perley, R. A., Chandler, C. J., Butler, B. J., \& Wrobel, J. M. 2011, ApJ (Letters), 739, L1

Peron, M. 2012, ASP Conf. Ser., 461, 115

Pesnell, W. D., Thompson, B. J., \& Chamberlin, P. C. 2012, Solar Phys., 275, 3

Pick, M. \& Vilmer, N. 2008, A\&ARv, 16, 1

Pietarila Graham, J., Cameron, R., \& Schüssler, M. 2010, ApJ, 714, 1606

Pietarila Graham, J., Danilovic, S., \& Schüssler, M. 2009, ApJ, 693, 1728

Puschmann, K. G., Denker, C., Kneer, F., Al Erdogan, N., Balthasar, H., Bauer, S. M., Beck, C., Bello González, N., Collados, M., Hahn, T., Hirzberger, J., Hofmann, A., Louis, R. E., Nicklas, H., Okunev, O., Martínez Pillet, V., Popow, E., Seelemann, T., Volkmer, R., Wittmann, A. D., \& Woche, M. 2012, Astron. Nachr., 333, 880

Puxley, P. \& Jørgensen, I. 2006, vol. 6270, SPIE Conf. Ser.

Radziszewski, K., Rudawy, P., \& Phillips, K. J. H. 2011, A\&A, 535, A123

Reardon, K. P. 2012, American Astronomical Society Meeting Abstracts, 220, \#323.05

Reardon, K. P., Rimmele, T., Tritschler, A., Cauzzi, G., Wöger, F., Uitenbroek, H., Tsuneta, S., \& Berger, T. 2009, ASP Conf. Ser., 415, 332

Rempel, M. 2011, ApJ, 729, 5

Richards, K., Rimmele, T., Hegwer, S. L., Upton, R. S., Wöger, F., Marino, J., Gregory, S., \& Goodrich, B. 2010, vol. 7736, SPIE Conf. Ser.

Rimmele, T. R., Keil, S., McMullin, J., Goode, P. R., Knölker, M., Kuhn, J. R., \& Rosner, R., ATST Team 2012, IAU Special Session, 6, E2.06

Rimmele, T. R., Keil, S., McMullin, J., Knölker, M., Kuhn, J. R., Goode, P. R., Rosner, R., Casini, R., Lin, H., Tritschler, A., \& Wöger, F., ATST Team 2012, ASP Conf. Ser., 463, 377 
Rimmele, T. R. \& Marino, J. 2011, Living Reviews in Solar Physics, 8, 2

Rimmele, T. R., Wöger, F., Marino, J., Richards, K., Hegwer, S., Berkefeld, T., Soltau, D., Schmidt, D., \& Waldmann, T. 2010, vol. 7736, SPIE Conf. Ser.

Sánchez Almeida, J. 2003, A\&BA, 411, 615

Schad, T. A., Jaeggli , S. A., Lin, H., \& Penn, M. J. 2011, ASP Conf. Ser., 437, 483

Schad, T. A., Penn, M. J., \& Lin, H. 2013, ApJ, 768, 111

Schad, T. A., Penn, M. J., Lin, H., \& Tritschler, A. 2012, ASP Conf. Ser., 463, 25

Scharmer, G. B., Löfdahl, M. G., van Werkhoven, T. I. M., \& de la Cruz Rodríguez, J. 2010, $A \mathscr{E} A, 521, \mathrm{~A} 68$

Scharmer, G. B., Narayan, G., Hillberg, T., de la Cruz Rodríguez, J., Löfdahl, M. G., Kiselman, D., Sütterlin, P., van Noort, M., \& Lagg, A. 2008, ApJ (Letters), 689, L69

Scharmer, G. B. \& van Werkhoven, T. I. M. 2010, A\&A, 513, A25

Schmidt, W., von der Lühe, O., Volkmer, R., Denker, C., Solanki, S. K., Balthasar, H., Bello Gonzalez, N., Berkefeld, T., Collados, M., Fischer, A., Halbgewachs, C., Heidecke, F., Hofmann, A., Kneer, F., Lagg, A., Nicklas, H., Popow, E., Puschmann, K. G., Schmidt, D., Sigwarth, M., Sobotka, M., Soltau, D., Staude, J., Strassmeier, K. G., \& Waldmann, T. A. 2012, Astron. Nachr., 333, 796

Schrijver, C. 2012, IAU Special Session, 6, E4.02

Schrijver, C. J., Title, A. M., Harvey, K. L., Sheeley, N. R., Wang, Y.-M., van den Oord, G. H. J., Shine, R. A., Tarbell, T. D., \& Hurlburt, N. E. 1998, Nature, 394, 152

Schüssler, M. \& Vögler, A. 2008, A\&A (Letters), 481, L5

Shimizu, T., Sakao, T., Katsukawa, Y., \& Group, J. S. W. 2012, ASP Conf. Ser., 454, 449

Silva, D. 2001, The Messenger, 105, 18

Skartlien, R. 2000, ApJ, 536, 465

Socas-Navarro, H. 2012, IAU Special Session, 6, E2.12

Socas-Navarro, H. 2005, ApJ (Letters), 633, L57

Socas-Navarro, H., Beckers, J., Brandt, P., Briggs, J., Brown, T., Brown, W., Collados, M., Denker, C., Fletcher, S., Hegwer, S., Hill, F., Horst, T., Komsa, M., Kuhn, J., Lecinski, A., Lin, H., Oncley, S., Penn, M., Rimmele, T., \& Streander, K. 2005, PASP, 117, 1296

Solanki, S. K., Barthol, P., Danilovic, S., Feller, A., Gandorfer, A., Hirzberger, J., Riethmüller, T. L., Schüssler, M., Bonet, J. A., Martínez Pillet, V., del Toro Iniesta, J. C., Domingo, V., Palacios, J., Knölker, M., Bello González, N., Berkefeld, T., Franz, M., Schmidt, W., \& Title, A. M. 2010, ApJ (Letters), 723, L127

Spruit, H. C., Title, A. M., \& van Ballegooijen, A. A. 1987, Solar Phys., 110, 115

Steiner, O. 2012, IAU Special Session, 6, E1.01

Steiner, O., Franz, M., Bello González, N., Nutto, C., Rezaei, R., Martínez Pillet, V., Bonet Navarro, J. A., del Toro Iniesta, J. C., Domingo, V., Solanki, S. K., Knölker, M., Schmidt, W., Barthol, P., \& Gandorfer, A. 2010, ApJ (Letters), 723, L180

Steiner, O. \& Rezaei, R. 2012, ASP Conf. Ser., 456, 3

Steiner, O., Rezaei, R., Schaffenberger, W., \& Wedemeyer-Böhm, S. 2008, ApJ (Letters), 680, L85

Stenflo, J. O. 1982, Solar Phys., 80, 209

Stenflo, J. O. 2010, A\&SA, 517, A37

Štěpán, J., Trujillo Bueno, J., Carlsson, M., \& Leenaarts, J. 2012, ApJ (Letters), 758, L43

Su, Y., Wang, T., Veronig, A., Temmer, M., \& Gan, W. 2012, ApJ (Letters), 756, L41

Suematsu, Y., Katsukawa, Y., Ichimoto, K., \& Shimizu, T. 2012, IAU Special Session, 6, E2.08

Tomczyk, S. 2012, IAU Special Session, 6, E2.14

Trujillo Bueno, J. 2012, IAU Special Session, 6, E3.02

Trujillo Bueno, J., Shchukina, N., \& Asensio Ramos, A. 2004, Nature, 430, 326

Trujillo Bueno, J., Štěpán, J., \& Belluzzi, L. 2012, ApJ (Letters), 746, L9

Tsuneta, S., Ichimoto, K., Katsukawa, Y., Lites, B. W., Matsuzaki, K., Nagata, S., Orozco Suárez, D., Shimizu, T., Shimojo, M., Shine, R. A., Suematsu, Y., Suzuki, T. K., Tarbell, T. D., \& Title, A. M. 2008, ApJ, 688, 1374

Uitenbroek, H. 2006, Organizations and Strategies in Astronomy, Vol. 7, 335, 117

Uitenbroek, H. \& Tritschler, A. 2012, IAU Special Session, 6, E4.01 
Uitenbroek, H. 2011, ASP Conf. Ser., 437, 439

Uitenbroek, H., Tritschler, A., \& Rimmele, T. 2007, ApJ, 668, 586

van Noort, M., Rouppe van der Voort, L., \& Löfdahl, M. G. 2005, Solar Phys., 228, 191

Vargas Domínguez, S., Palacios, J., Balmaceda, L., Cabello, I., \& Domingo, V. 2011, MNRAS, 416,148

Viticchié, B., Sánchez Almeida, J., Del Moro, D., \& Berrilli, F. 2011, A\&A, 526, A60

Vögler, A. \& Schüssler, M. 2007, A\&A (Letters), 465, L43

Wang, J., Jin, C., \& Zhou, G. 2012, IAU Special Session, 6, E1.08

Wedemeyer, S., Scullion, E., Steiner, O., de la Cruz Rodriguez, J., \& Rouppe van der Voort, L. 2013, arXiv1303.0179

Wedemeyer-Böhm, S., Ludwig, H.-G., Steffen, M., Freytag, B., \& Holweger, H. 2005, ESA Special Publication, 560, 1035

Wedemeyer-Böhm, S. \& Rouppe van der Voort, L. 2009, A\&\&A, 503, 225

Wedemeyer-Böhm, S. \& Rouppe van der Voort, L. 2009, A\&A, 507, L9

Wedemeyer-Böhm, S., Scullion, E., Steiner, O., Rouppe van der Voort, L., de La Cruz Rodriguez, J., Fedun, V., \& Erdélyi, R. 2012, Nature, 486, 505

Wiegelmann, T., Solanki, S. K., Borrero, J. M., Martínez Pillet, V., del Toro Iniesta, J. C., Domingo, V., Bonet, J. A., Barthol, P., Gandorfer, A., Knölker, M., Schmidt, W., \& Title, A. M. 2010, ApJ (Letters), 723, L185

Wöger, F. 2010, Applied Optics, 49, 1818

Xu, Y., Cao, W., Jing, J., \& Wang, H. 2012, ApJ (Letters), 750, L7

Yan, Y., Zhang, J., Chen, Z., Wang, W., Liu, F., \& Geng, L. 2012, IAU Special Session, 6, E2.16

Yan, Y., Zhang, J., Wang, W., Liu, F., Chen, Z., \& Ji, G. 2009, Earth Moon and Planets, 104, 97

Yelles Chaouche, L., Moreno-Insertis, F., Martínez Pillet, V., Wiegelmann, T., Bonet, J. A., Knölker, M., Bellot Rubio, L. R., del Toro Iniesta, J. C., Barthol, P., Gandorfer, A., Schmidt, W., \& Solanki, S. K. 2011, ApJ (Letters), 727, L30

Zhang, J. \& Liu, Y. 2011, ApJ (Letters), 741, L7

\section{Appendix: Program of Special Session 6 at IAU XXVIII General Assembly}

\section{SESSION 1 - KEY SCIENTIFIC QUESTIONS}

OVERVIEW:

Oskar Steiner: Science challenges for large solar telescopes

STATE OF THE ART:

Dali Georgobiani, R. Stein, A. Nordlund: Realistic numerical simulations of solar convection: emerging flux, pores, and Stokes spectra

Ryohko Ishikawa: Properties of transient horizontal magnetic fields, and their implication for the origin of quiet-Sun magnetism

Irina Kitiashvili. et al. Investigation of small scale turbulent MHD phenomena using numerical simulations and NST observations

Juan Martínez-Sykora (Metcalf Lecturer): Current status of self-consistent 3D radiativeMHD simulations of the solar atmosphere

Phil Judge and R. Casini: Using large telescopes to answer: why must the Sun have a chromosphere and corona?

James Klimchuck: The role of spicules in explaining the corona and the transition region 
Jingxiu Wang et al.: Solar intranetwork magnetic elements - the weakest component of solar magnetism

\section{SESSION 2 - EXISTING AND PLANNED LARGE FACILITIES: WHAT IS THEIR IMPACT ON KEY SCIENTIFIC QUESTIONS?}

\section{OVERVIEW:}

Thomas Berger: Science with large solar telescopes: Addressing key science questions with new observing modes

\section{NEWLY OPERATED FACILITIES:}

Wenda Cao: The $1.6 \mathrm{~m}$ New Solar Telescope (NST) in Big Bear

Carsten Denker et al.: The GREGOR Solar Telescope

Haisheng Ji: The one meter aperture solar telescope in China

Arnold Benz: Observing the Sun with ALMA

\section{UPCOMING FACILITIES:}

Thomas Rimmele et al. (presented by T. Berger): Construction of the Advanced Technology Solar Telescope - a progress report

Yukio Katsukawa et al.: Next space solar observatory SOLAR-C: mission overview and science objectives

Yoshinori Suematsu et al.: Science and instrument design of $1.5 \mathrm{~m}$ aperture Solar Optical Telescope for the Solar-C mission

Yuanyong Deng (on behalf of the SST group): The Space Solar Telescope

Sergey Bogachev et al.: The set of imaging instruments for Interhelioprobe solar observatory

Shiraj Hasan: The National Large Solar Telescope (NLST) of India

Hector Socas Navarro: The European Solar Telescope

Zhong Liu et al.: An introduction to the Chinese Giant Solar Telescope

Steve Tomzcyk: The Coronal Solar Magnetism Observatory

Tim Bastian (delivered by A. Benz): Observing the Sun at radio-wavelengths: current status and future prospects

Yihua Yan et al.: On solar radio imaging-spectroscopy

SESSION 3 - STEP AHEAD: FUTURE SCIENCE AND WHAT THE NEW FACILITIES SHOULD NOT FORGET !

Marianne Faurobert: The quiet Sun magnetism: What can we learn from the Hanle effect? Javier Trujillo Bueno: Polarized Radiation Diagnostics for Measuring the Magnetic Field of the Outer Solar Atmosphere

Valentin Martínez Pillet: Towards the next frontier in high precision solar polarimetry: $10^{-4}$

Mats Löfdahl: Restoration of the contrast in solar images

MingDe Ding: Spectral diagnostics of the heating and dynamics of the solar chromosphere Na Deng: H $\alpha$ Imaging Spectroscopy of a C-class Flare with IBIS

\section{SESSION 4 - LESSON LEARNED, AND FUTURE DIRECTIONS FOR TELESCOPE OPERATION}

Han Uitenbroek, A. Tritschler: Observing strategies for future solar facilities: the ATST test case

Karel Schrjiver: Mining Solar Data: the experience with SDO, Hinode, and TRACE

\section{GENERAL DISCUSSION}


POSTERS:

A. Benz et al.: Spectrometer Telescope for Imaging X-rays (STIX)

L. Geng: The 0.4-2.0GHz Analogous Receiving System Performance of CSRH

S. Li et al.: Radiation pattern measurement for Chinese Spectral Radio Heliograph

A. Hady: Geomagnetic storms in the rising phase of solar cycle 24

A. Valio et al.: Solar Patrol Polarization Telescopes at 45 and $90 \mathrm{GHz}$

Y. Sun: The Measuring System of Near Infrared Fabry-Perot Etalon

J. Lin, Y. Deng: Automatic full disk vector magnetogram observing system in HSOS

$J$. Lin et al.: Research about the high precision temperature measurement

J. Guo: A statistical study on the soft X-ray flare in solar cycles 22 and 23

W. P. Zhou, Y. Wang: A new energy-efficient control approach for astronomical telescope drive system

W. P. Zhou, Y. Wang: The real-time motion control with high precision for the groundbased telescopes

F. Ibodov, S. Ibadov: High-resolution observations of solar explosive phenomena due to cometary impacts with the Sun

G. Cauzzi, K. Reardon: The IBIS Mosaic 